\title{
Expected Values of Molecular Descriptors in Random Polyphenyl Chains
}

\author{
Zahid Raza ${ }^{1 *}$, Kiran Naz ${ }^{2}$, Sarfraz Ahmad ${ }^{2}$ \\ ${ }^{1}$ Department of Mathematics, College of Sciences, University of Sharjah, UAE. \\ ${ }^{2}$ Department of Mathematics, COMSATS University Islamabad, Lahore Campus Pakistan.
}

\begin{abstract}
A chemical graph is a model used to indicate a chemical combination. In a molecular graph, vertices define atoms, and edges are represented as chemical bonds. A topological index is a single number to characterize the graph of a molecule. In this article, we study the topological properties of some special chains. The polyphenyl chains with hexagons are graphs of aromatic organic compounds. The key purpose of this article is to explore the expected value of Sombor, reduced Sombor, and average Sombor index for this category of organic compounds. It was investigated that the Sombor, reduced Sombor and average Sombor index revealed adequate discriminative potential of alkanes. It has been tested that these indicators can be used effectively in modeling chemical thermodynamic structures. The average value of the Sombor, reduced Sombor, and average Sombor index for the set of all spiro and random polyphenyl chains has been determined Finally, the ratio between the expected values of these mentioned indices for both random chains has been resolved.
\end{abstract}

\section{Keywords:}

Sombor Index;

Reduced Sombor Index;

Average Sombor Index;

Spiro Chain; Polyphenyl Chains.

\section{Article History:}

Received: 19 September 2021

Revised: $\quad 25 \quad$ November 2021

Accepted: 27 December 2021

Published: $01 \quad$ February 2022

\section{1- Introduction}

Aromatic organic compounds are main building blocks for many natural and synthetic chemical compounds as well as constituents of petrochemicals. Topological index was first created in 1971 by Hossoya. The simplest topological index does not acknowledge double bonds in hydrocarbons. Polycyclic aromatic hydrocarbons are well studied and much explored chemicals in food. Polyphenols are plant defense system (secondary metabolites) originate in vegetables, fruits and seeds. Polyphenols have diversity in their chemical structure along with a wide range in their properties and applications like as natural antioxidants, cardiovascular inflammatory, and neurodegenerative diseases, food supplements, pharmaceutical and cosmetic additive. These days, there are many topological indicators [1], some of which are incorporated into chemistry. They can be distinguished by the structure of the graphs used for their calculation. For example, there is a Hosoya index, which is calculated by counting non-incident edges on a graph. In addition, the Estrada index is based on graph width, the Randic link index, and the Zagreb group indicators are calculated using degrees of vertices, etc. Topological indices have many applications in QSPR/QSAR research. The degree based topological index play an important part in chemical graph theory. The Sombor index [2-4] family was introduced by Gutman with a view to a chemical graph. In this article we are concerned about Sombor's index of chemical graphs. Topological indices are related to vertex adjacency or the topological distances in a graph. Wiener index [5-7] is the first topological index, which is equal to the sum of all distances between the vertices. In this article, we will compute the topological index like $S O, S O_{\text {red }}$ and $S O_{\text {avg }}$. The $S O, S O_{\text {red }}$ and $S O_{\text {avg }}[8]$ are defined as:

*CONTACT: zraza@sharjah.ac.ae

DOI: http://dx.doi.org/10.28991/ESJ-2022-06-01-012

(C) 2022 by the authors. Licensee ESJ, Italy. This is an open access article under the terms and conditions of the Creative Commons Attribution (CC-BY) license (https://creativecommons.org/licenses/by/4.0/). 
$\mathrm{SO}(\mathrm{G})=\sum_{\mathrm{e}_{\mathrm{i}, \mathrm{j}} \mathrm{E}(\mathrm{G})} \sqrt{\operatorname{deg}\left(\mathrm{v}_{\mathrm{i}}\right)^{2}+\operatorname{deg}\left(\mathrm{v}_{\mathrm{j}}\right)^{2}}$

$\mathrm{SO}_{\text {red }}(\mathrm{G})=\sum_{\mathrm{e}_{\mathrm{i}, \mathrm{j}} \mathrm{E}(\mathrm{G})} \sqrt{\operatorname{deg}\left(\left(\mathrm{v}_{\mathrm{i}}\right)-1\right)^{2}+\operatorname{deg}\left(\left(\mathrm{v}_{\mathrm{j}}\right)-1\right)^{2}}$

$\mathrm{SO}_{\mathrm{avg}}(\mathrm{G})=\sum_{\mathrm{e}_{\mathrm{i}, \mathrm{j}} \in \mathrm{E}(\mathrm{G})} \sqrt{\left(\operatorname{deg}\left(\mathrm{v}_{\mathrm{i}}\right)-\frac{2 \mathrm{~m}}{\mathrm{n}}\right)^{2}+\left(\operatorname{deg}\left(\mathrm{v}_{\mathrm{j}}\right)-\frac{2 \mathrm{~m}}{\mathrm{n}}\right)^{2}}$

where $\mathrm{m}$ denotes the number of edges and $\mathrm{n}$ denotes the number of vertices. Let us consider hexagons $\hbar \_1, \hbar \_2, \hbar 33, \ldots ., \hbar \_$k. We can get a Polyphenyl chains $(\hat{R} P C)$ by adding an edge to each pair of successive hexagons [9]. Since two successive hexagons can be attached by three different schemes as shown in Figure 1 . For $k>2$, random Polyphenyl chains $\left(\hat{\mathrm{R}} P C_{k}\right)$ may not be unique. The three types of order denoted by $\mathrm{R} P C_{k}^{1}$, $\hat{\mathrm{R}} P C_{k}^{2}$, and $\hat{\mathrm{R}} P C_{k}^{3}$. Let us associate the probabilities $\zeta_{1}, \zeta_{2}$ and $1-\zeta_{1}-\zeta_{2}$ for acquiring $\hat{\mathrm{R}} P C_{k}^{1}, \hat{\mathrm{R}} P C_{k}^{2}$, and $\hat{\mathrm{R}} P C_{k}^{3}$.

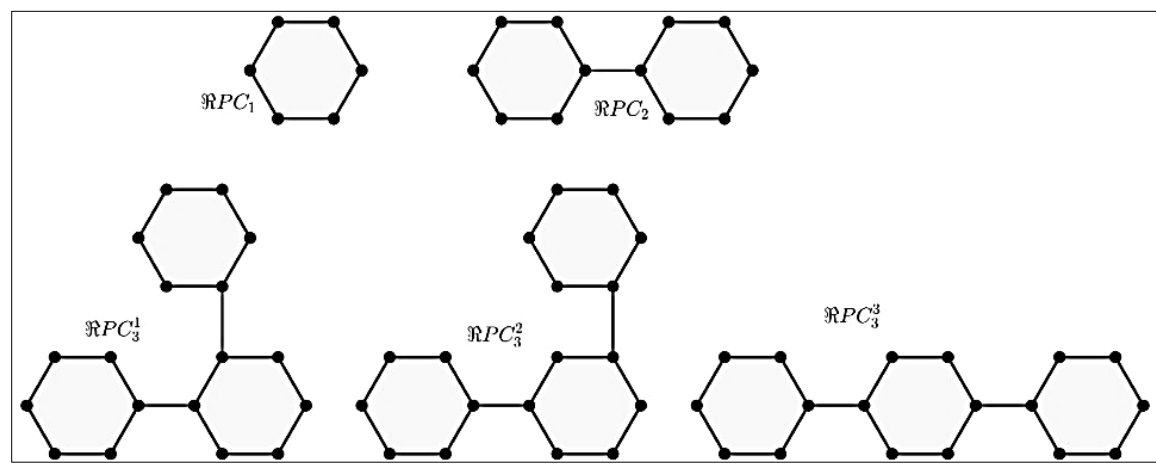

Figure 1. Polyphenyl Chains

If we squeeze each bridge between successive hexagons in ( $\left.R P C_{k}\right)$, after that we will get a spiro-chain which is symbolized by $\left(\hat{\mathrm{R}} P C_{k}\right)$. For $k>2$, spiro chain $[6,10,11]$ is not unique and has following three arrangements as shown in Figure 2, denoted by R $P C_{k}^{1}, \hat{R} P C_{k}^{2}$, and $\hat{R} P C_{k}^{3}$. Let us link the probabilities $\zeta_{1}, \zeta_{2}$ and $1-\zeta_{1}-\zeta_{2}$ for acquiring $\hat{\mathrm{R}} P C_{k}^{1}, \mathrm{R} P C_{k}^{2}$, and $\hat{\mathrm{R}} P C_{k}^{3}$.

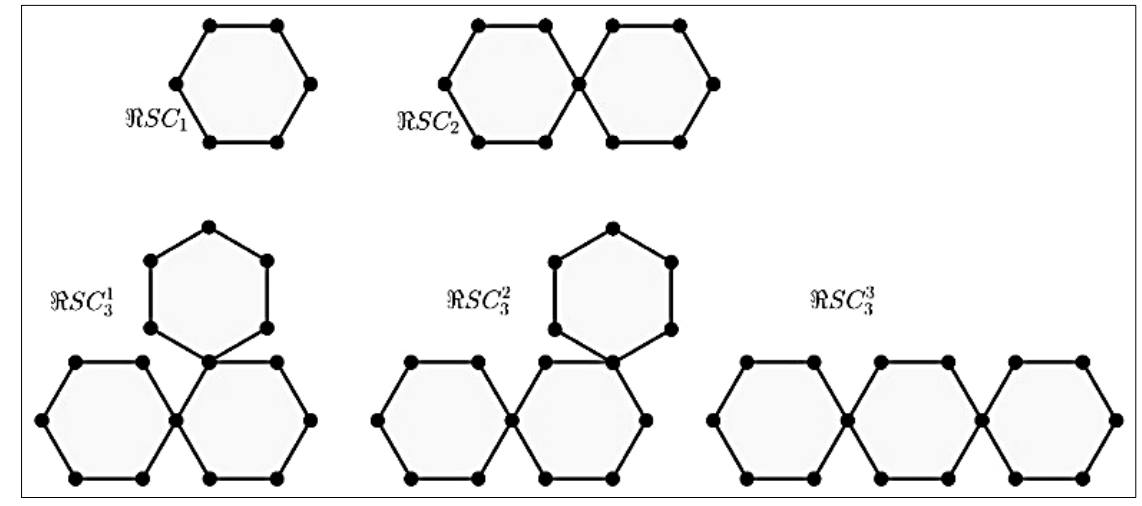

Figure 2. Spiro Chain

\section{2- Sombor, Reduced Sombor and Average Sombor in Random Polyphenyl Chains}

In this part, we examine the $S O, S O_{r e d}$ and $S O_{a v g}$ in the random Polyphenyl chains $R P C_{k}$ Let $R P C_{k}$ be the polyphenyl chain [12] obtained by ŔP $C_{k-1}$. Clearly, there are only $(2,2),(2,3)$ and $(3,3)$ - edges in $R C_{k}$ By using the Equations 1 and 2 and Equation 3 in random Polyphenyl chains [13] we have the following equations:

$$
\begin{aligned}
& \mathrm{SO}\left(\mathrm{ŔPC}_{\mathrm{k}}\right)=2 \sqrt{2} \omega_{22}\left(\mathrm{ŔPC}_{\mathrm{k}}\right)+\sqrt{13} \omega_{23}\left(\mathrm{ŔPC}_{\mathrm{k}}\right)+3 \sqrt{2} \omega_{33}\left(\mathrm{ŔPC}_{\mathrm{k}}\right) \\
& \mathrm{SO}_{\text {red }}\left(\mathrm{ŔPC}_{\mathrm{k}}\right)=\sqrt{2} \omega_{22}\left(\mathrm{ŔPC}_{\mathrm{k}}\right)+\sqrt{5} \omega_{23}\left(\mathrm{ŔPC}_{\mathrm{k}}\right)+2 \sqrt{2} \omega_{33}\left(\mathrm{Ŕ}_{\mathrm{k}}\right) \\
& \mathrm{SO}_{\text {avg }}\left(\operatorname{Ŕ}^{\mathrm{P}} \mathrm{k}_{\mathrm{k}}\right)=\sqrt{2}\left(\frac{\mathrm{k}-1}{3 \mathrm{k}}\right) \omega_{22}\left(\mathrm{Ŕ}_{\mathrm{k}}\right)+\frac{\sqrt{5 \mathrm{k}^{2}+2 \mathrm{k}+2}}{3 \mathrm{k}} \omega_{23}\left(\mathrm{ŔPC}_{\mathrm{k}}\right)+\sqrt{2}\left(\frac{2 \mathrm{k}+1}{3 \mathrm{k}}\right) \omega_{33}\left(\mathrm{Ŕ}_{\mathrm{k}}\right)
\end{aligned}
$$


Thus, to find the $S O, S O_{\text {red }}$ and $S O_{a v g}$ of $R P C_{k}$, we just need to evaluate the $\omega_{22}\left(\hat{\mathrm{R}} P C_{k}\right), \omega_{23}\left(\hat{\mathrm{R}} P C_{k}\right)$ and $\omega_{23}\left(\hat{\mathrm{R}} P C_{k}\right)$. Since $\mathrm{R} P C_{k}\left(k, \zeta_{1}, \zeta_{2}\right)$ is a random Polyphenyl chains. So, $S O\left(\hat{\mathrm{R}} P C_{k}\left(k, \zeta_{1}, \zeta_{2}\right)\right)$, $S O_{\text {red }}\left(\hat{\mathrm{R}} P C_{k}\left(k, \zeta_{1}, \zeta_{2}\right)\right)$ and $S O_{\text {avg }}\left(\hat{\mathrm{R}} P C_{k}\left(k, \zeta_{1}, \zeta_{2}\right)\right)$ are also arbitrary variables. We symbolize the anticipated values of given indices by $E_{k}^{S O}=E^{S O}\left[S O\left(\hat{\mathrm{R}} P C_{k}\left(k, \zeta_{1}, \zeta_{2}\right)\right)\right], E_{k}^{\text {red }}=E^{\text {red }}\left[S O\left(\hat{\mathrm{R}} P C_{k}\left(k, \zeta_{1}, \zeta_{2}\right)\right)\right]$ and $E_{k}^{\text {avg }}=E^{\text {avg }}\left[S O\left(\hat{\mathrm{R}} P C_{k}\left(k, \zeta_{1}, \zeta_{2}\right)\right)\right]$ respectively.

\section{2-1-Theorem}

Let $\mathrm{R} P C_{k}\left(k, \zeta_{1}, \zeta_{2}\right)$ be an arbitrary Polyphenyl chains of length $k$, where $k \geq 2$. Then: $\mathrm{E}_{\mathrm{k}}^{\mathrm{SO}}=\left[(5 \sqrt{2}-2 \sqrt{13}) \zeta_{1}+(7 \sqrt{2}+4 \sqrt{13})\right] \mathrm{k}-(10 \sqrt{2}-4 \sqrt{13}) \zeta_{1}+(5 \sqrt{2}-4 \sqrt{13})$

Proof: For $k=2$, we have $E_{2}^{S O}=19 \sqrt{2}+4 \sqrt{13}$. Now, when $k \geq 2$, it is obvious that $\omega_{22}, \omega_{23}$ and $\omega_{33}$ depend on the following three cases:

1. If $\hat{\mathrm{R}} P C_{k-1} \rightarrow \mathrm{R} P C_{k}^{1}$ with probability $\zeta_{1}$

$\omega_{22}\left(\operatorname{ŔPC}_{\mathrm{k}}^{1}\right)=\omega_{22}\left(\mathrm{Ŕ}_{\mathrm{PC}-1}\right)+3$

$\omega_{23}\left(\operatorname{ŔPC}_{\mathrm{k}}^{1}\right)=\omega_{23}\left(\mathrm{ŔPC}_{\mathrm{k}-1}\right)+2$

$\omega_{33}\left(\mathrm{Ŕ}_{\mathrm{k}}^{1}\right)=\omega_{33}\left(\mathrm{ŔPC}_{\mathrm{k}-1}\right)+2$

by using Equation 4, we have:

$\mathrm{SO}\left(\mathrm{ŔPC}_{\mathrm{k}}^{1}\right)=\mathrm{SO}\left(\mathrm{ŔPC}_{\mathrm{k}-1}\right)+12 \sqrt{2}+2 \sqrt{13}$

2. If $\hat{\mathrm{R}} P C_{k-1} \rightarrow \hat{\mathrm{R}} P C_{k}^{2}$ with probability $\zeta_{2}$

$\omega_{22}\left(\hat{\mathrm{R}} P C_{k}^{2}\right)=\omega_{22}\left(\hat{\mathrm{R}} P C_{k-1}\right)+2$

$\omega_{23}\left(\hat{\mathrm{R}} P C_{k}^{2}\right)=\omega_{23}\left(\hat{\mathrm{R}} P C_{k-1}\right)+4$

$\omega_{33}\left(\hat{\mathrm{R}} P C_{k}^{2}\right)=\omega_{33}\left(\hat{\mathrm{R}} P C_{k-1}\right)+1$

by using Equation 4, we have:

$\mathrm{SO}\left(\mathrm{ŔPC}_{\mathrm{k}}^{2}\right)=\mathrm{SO}\left(\mathrm{ŔPC}_{\mathrm{k}-1}\right)+7 \sqrt{2}+4 \sqrt{13}$

3. If $\mathrm{R} P C_{k-1} \rightarrow \hat{\mathrm{R}} P C_{k}^{3}$ with probability $1-\zeta_{1}-\zeta_{2}$

$\omega_{22}\left(\mathrm{ŔPC}_{\mathrm{k}}^{3}\right)=\omega_{22}\left(\mathrm{ŔPC}_{\mathrm{k}-1}\right)+2$

$\omega_{23}\left(\mathrm{ŔPC}_{\mathrm{k}}^{3}\right)=\omega_{23}\left(\mathrm{ŔPC}_{\mathrm{k}-1}\right)+4$

$\omega_{33}\left(\operatorname{ŔPC}_{\mathrm{k}}^{3}\right)=\omega_{33}\left(\mathrm{ŔPC}_{\mathrm{k}-1}\right)+1$

by using Equation 4, we have:

$\mathrm{SO}\left(\mathrm{ŔPC}_{\mathrm{k}}^{3}\right)=\mathrm{SO}\left(\mathrm{ŔPC}_{\mathrm{k}-1}\right)+7 \sqrt{2}+4 \sqrt{13}$

Thus:

$\mathrm{E}_{\mathrm{k}}^{\mathrm{SO}}=\zeta_{1} \mathrm{SO}\left(\mathrm{ŔPC}_{\mathrm{k}}^{1}\right)+\zeta_{2} \mathrm{SO}\left(\mathrm{ŔPC}_{\mathrm{k}}^{2}\right)+\left(1-\zeta_{1}-\zeta_{2}\right) \mathrm{SO}\left(\mathrm{ŔPC}_{\mathrm{k}}^{3}\right)$

$\left.\mathrm{E}_{\mathrm{k}}^{\mathrm{SO}}=\zeta_{1} \mathrm{SO}\left(\mathrm{ŔPC}_{\mathrm{k}-1}\right)+(12 \sqrt{2}+2 \sqrt{13}) \zeta_{1}+\zeta_{2} \mathrm{SO}_{(\mathrm{R} P C}-1\right)+(7 \sqrt{2}+4 \sqrt{13}) \zeta_{2}+\left(1-\zeta_{1}-\zeta_{2}\right) \mathrm{SO}_{\mathrm{k}}\left(\mathrm{Ŕ}_{\mathrm{k}}^{3}\right)+$ $\left(1-\zeta_{1}-\zeta_{2}\right)(12 \sqrt{2}+2 \sqrt{13})$

$\mathrm{E}_{\mathrm{k}}^{\mathrm{SO}}=\mathrm{SO}\left(\mathrm{ŔPC}_{\mathrm{k}-1}\right)+(5 \sqrt{2}-2 \sqrt{13}) \zeta_{1}+(7 \sqrt{2}+4 \sqrt{13})$

Since $E\left[E_{k}\right]^{S O}=E_{k}^{S O}$ so apply the operator $E$ on Equation 7, we get:

$\mathrm{E}_{\mathrm{k}}^{\mathrm{SO}}=\mathrm{E}_{\mathrm{k}-1}^{\mathrm{SO}}+(5 \sqrt{2}-2 \sqrt{13}) \zeta_{1}+(7 \sqrt{2}+4 \sqrt{13}), \quad \mathrm{k}>2$

and after solving the recurrence relation in Equation 8 with $E_{2}^{S O}=19 \sqrt{2}+4 \sqrt{13}$, we get

$\mathrm{E}_{\mathrm{k}}^{\mathrm{SO}}=\left[(5 \sqrt{2}-2 \sqrt{13}) \zeta_{1}+(7 \sqrt{2}+4 \sqrt{13})\right] \mathrm{k}-(10 \sqrt{2}-4 \sqrt{13}) \zeta_{1}+(5 \sqrt{2}-4 \sqrt{13})$.

\section{2-2-Theorem}

Let $\hat{\mathrm{R}} P C_{k}\left(k, \zeta_{1}, \zeta_{2}\right)$ be an arbitrary Polyphenyl chains of length $k$, where $k \geq 2$. Then $\mathrm{E}_{\mathrm{k}}^{\mathrm{red}}=\left[(3 \sqrt{2}-2 \sqrt{5}) \zeta_{1}+(4 \sqrt{2}+4 \sqrt{5})\right] \mathrm{k}-(6 \sqrt{2}-4 \sqrt{5}) \zeta_{1}+(2 \sqrt{2}-4 \sqrt{5})$. 
Proof: For $k=2$, we have $E_{2}^{\text {red }}=10 \sqrt{2}+4 \sqrt{5}$. Now, when $k \geq 3$, it is obvious that $\omega_{22}, \omega_{23}$ and $\omega_{33}$ depend on the following three cases:

If $\hat{\mathrm{R}} P C_{k-1} \rightarrow \hat{\mathrm{R}} P C_{k}^{1}$ with probability $\zeta_{1}$

$\omega_{22}\left(\mathrm{Ŕ}_{\mathrm{k}}^{1}\right)=\omega_{22}\left(\mathrm{ŔPC}_{\mathrm{k}-1}\right)+3$

$\omega_{23}\left(\mathrm{Ŕ}_{\mathrm{k}}^{1}\right)=\omega_{23}\left(\mathrm{ŔPC}_{\mathrm{k}-1}\right)+2$

$\omega_{33}\left(\operatorname{Ŕ}_{\mathrm{k}}^{1}\right)=\omega_{33}\left(\mathrm{ŔPC}_{\mathrm{k}-1}\right)+2$

by using Equation 5, we have:

$\mathrm{SO}_{\text {red }}\left(\mathrm{ŔPC}_{\mathrm{k}}^{1}\right)=\mathrm{SO}_{\text {red }}\left(\mathrm{ŔPC}_{\mathrm{k}-1}\right)+7 \sqrt{2}+2 \sqrt{5}$

1. If $\mathrm{R} P C_{k-1} \rightarrow \hat{\mathrm{R}} P C_{k}^{2}$ with probability $\zeta_{2}$

$\omega_{22}\left(\hat{\mathrm{R}} P C_{k}^{2}\right)=\omega_{22}\left(\hat{\mathrm{R}} P C_{k-1}\right)+2$

$\omega_{23}\left(\hat{\mathrm{R}} P C_{k}^{2}\right)=\omega_{23}\left(\hat{\mathrm{R}} P C_{k-1}\right)+4$

$\omega_{33}\left(\mathrm{R} P C_{k}^{2}\right)=\omega_{33}\left(\mathrm{R} P C_{k-1}\right)+1$

by using Equation 5, we have:

$\mathrm{SO}_{\text {red }}\left(\mathrm{ŔPC}_{\mathrm{k}}^{2}\right)=\mathrm{SO}_{\text {red }}\left(\mathrm{ŔPC}_{\mathrm{k}-1}\right)+4 \sqrt{2}+4 \sqrt{5}$

1. If $\hat{\mathrm{R}} P C_{k-1} \rightarrow \mathrm{R} P C_{k}^{3}$ with probability $1-\zeta_{1}-\zeta_{2}$

$\omega_{22}\left(\hat{\mathrm{R}} P C_{k}^{3}\right)=\omega_{22}\left(\hat{\mathrm{R}} P C_{k-1}\right)+2$

$\omega_{23}\left(\hat{\mathrm{R}} P C_{k}^{3}\right)=\omega_{23}\left(\hat{\mathrm{R}} P C_{k-1}\right)+4$

$\omega_{33}\left(\hat{\mathrm{R}} P C_{k}^{3}\right)=\omega_{33}\left(\hat{\mathrm{R}} P C_{k-1}\right)+1$

by using Equation 5, we have:

$\mathrm{SO}_{\text {red }}\left(\mathrm{ŔPC}_{\mathrm{k}}^{3}\right)=\mathrm{SO}_{\text {red }}\left(\mathrm{ŔPC}_{\mathrm{k}-1}\right)+4 \sqrt{2}+4 \sqrt{5}$

Thus:

$\mathrm{E}_{\mathrm{k}}^{\text {red }}=\zeta_{1} \mathrm{SO}_{\text {red }}\left(\mathrm{ŔPC}_{\mathrm{k}}^{1}\right)+\zeta_{2} \mathrm{SO}_{\text {red }}\left(\mathrm{ŔPC}_{\mathrm{k}}^{2}\right)+\left(1-\zeta_{1}-\zeta_{2}\right) \mathrm{SO}_{\text {red }}\left(\mathrm{ŔPC}_{\mathrm{k}}^{3}\right)$

$\mathrm{E}_{\mathrm{k}}^{\mathrm{red}}=\zeta_{1} \mathrm{SO}_{\text {red }}\left(\mathrm{R} P C_{\mathrm{k}-1}\right)+(7 \sqrt{2}+2 \sqrt{5}) \zeta_{1}+\zeta_{2} \mathrm{SO}_{\text {red }}\left(\mathrm{ŔPC}_{\mathrm{k}-1}\right)+(4 \sqrt{2}+4 \sqrt{5}) \zeta_{2}+\left(1-\zeta_{1}-\zeta_{2}\right) \mathrm{SO}_{\text {red }}\left(\mathrm{R} P C_{\mathrm{k}}^{3}\right)+$ $\left(1-\zeta_{1}-\zeta_{2}\right)(4 \sqrt{2}+2 \sqrt{5})$

$\mathrm{E}_{\mathrm{k}}^{\text {red }}=\mathrm{SO}_{\text {red }}\left(\mathrm{ŔPC}_{\mathrm{k}-1}\right)+(3 \sqrt{2}-2 \sqrt{13}) \zeta_{1}+(4 \sqrt{2}+4 \sqrt{5})$

Since $E\left[E_{k}\right]^{\text {red }}=E_{k}^{\text {red }}$ so apply the operator $E$ on Equation 9, we get:

$\mathrm{E}_{\mathrm{k}}^{\mathrm{red}}=\mathrm{E}_{\mathrm{k}-1}^{\mathrm{red}}+(3 \sqrt{2}-2 \sqrt{5}) \zeta_{1}+(4 \sqrt{2}+4 \sqrt{5}), \mathrm{k}>2$

And after solving the recurrence relation in Equation 10 with $E_{2}^{\text {red }}=10 \sqrt{2}+4 \sqrt{5}$, we get:

$\mathrm{E}_{\mathrm{k}}^{\mathrm{red}}=\left[(3 \sqrt{2}-2 \sqrt{5}) \zeta_{1}+(4 \sqrt{2}+4 \sqrt{5})\right] \mathrm{k}-(6 \sqrt{2}-4 \sqrt{5}) \zeta_{1}+(2 \sqrt{2}-4 \sqrt{5})$.

\section{2-3-Theorem}

Let $\mathrm{R} P C_{k}\left(k, \zeta_{1}, \zeta_{2}\right)$ be an arbitrary Polyphenyl chains of length $k$, where $k \geq 2$. Then

$\mathrm{E}_{\mathrm{k}}^{\mathrm{avg}}=\left[\left(\sqrt{2}-\frac{2 \sqrt{5 \mathrm{k}^{2}+2 \mathrm{k}+2}}{3 \mathrm{k}}\right) \zeta_{1}+\left(\frac{4 \sqrt{2} \mathrm{k}-\sqrt{2}}{3 \mathrm{k}}+\frac{4 \sqrt{5 \mathrm{k}^{2}+2 \mathrm{k}+2}}{3 \mathrm{k}}\right)\right] \mathrm{k}-\left(2 \sqrt{2}-\frac{4 \sqrt{5 \mathrm{k}^{2}+2 \mathrm{k}+2}}{3 \mathrm{k}}\right) \zeta_{1}-\left(\frac{8 \sqrt{2} \mathrm{k}-2 \sqrt{2}}{3 \mathrm{k}}+\frac{8 \sqrt{5 \mathrm{k}^{2}+2 \mathrm{k}+2}}{3 \mathrm{k}}\right)+\frac{13 \sqrt{2}}{6}+\frac{2 \sqrt{26}}{3}$.

Proof: For $k=2$, we have $E_{2}^{a v g}=\frac{13 \sqrt{2}}{6}+\frac{2 \sqrt{26}}{3}$. Now, when $k \geq 3$, it is obvious that $\omega_{22}, \omega_{23}$ and $\omega_{33}$ depend on the following three cases:

1. If Ŕ $P C_{k-1} \rightarrow R ́ R C_{k}^{1}$ with probability $\zeta_{1}$

$\omega_{22}\left(\hat{R}^{\prime} C_{\mathrm{k}}^{1}\right)=\omega_{22}\left(\hat{R}^{\prime} C_{\mathrm{k}-1}\right)+3$

$\omega_{23}\left(\mathrm{Ŕ}_{\mathrm{k}}^{1}\right)=\omega_{23}\left(\mathrm{ŔPC}_{\mathrm{k}-1}\right)+2$

$\omega_{33}\left(\operatorname{ŔPC}_{\mathrm{k}}^{1}\right)=\omega_{33}\left(\mathrm{ŔPC}_{\mathrm{k}-1}\right)+2$ 
by using Equation 5, we have:

$\mathrm{SO}_{\mathrm{avg}}\left(\mathrm{RPC}_{\mathrm{k}}^{1}\right)=\mathrm{SO}_{\mathrm{avg}}\left(\mathrm{R} P C_{\mathrm{k}-1}\right)+\left(\frac{7 \sqrt{2} \mathrm{k}-\sqrt{2}}{3 \mathrm{k}}\right)+\left(\frac{2 \sqrt{5 \mathrm{k}^{2}+2 \mathrm{k}+2}}{3 \mathrm{k}}\right)$

2. If $\mathrm{R} P C_{k-1} \rightarrow \mathrm{R} P C_{k}^{2}$ with probability $\zeta_{2}$

$\omega_{22}\left(\mathrm{Ŕ}_{\mathrm{P}}^{2}\right)=\omega_{22}\left(\mathrm{ŔPC}_{\mathrm{k}-1}\right)+2$

$\left(\mathrm{ŔPC}_{\mathrm{k}}^{2}\right)=\omega_{23}\left(\mathrm{ŔPC}_{\mathrm{k}-1}\right)+4$

$\omega_{33}\left(\operatorname{ŔPC}_{\mathrm{k}}^{2}\right)=\omega_{33}\left(\mathrm{ŔPC}_{\mathrm{k}-1}\right)+1$

by using Equation 5, we have:

$\mathrm{SO}_{\mathrm{avg}}\left(\mathrm{R}^{\mathrm{P}} \mathrm{C}_{\mathrm{k}}^{2}\right)=\mathrm{SO}_{\mathrm{avg}}\left(\mathrm{ŔPC}_{\mathrm{k}-1}\right)+\left(\frac{4 \sqrt{2} \mathrm{k}-\sqrt{2}}{3 \mathrm{k}}\right)+\left(\frac{4 \sqrt{5 \mathrm{k}^{2}+2 \mathrm{k}+2}}{3 \mathrm{k}}\right)$

3. If $\mathrm{R} P C_{k-1} \rightarrow \mathrm{R} P C_{k}^{3}$ with probability $1-\zeta_{1}-\zeta_{2}$

$\omega_{22}\left(\mathrm{Ŕ}_{\mathrm{k}}^{3}\right)=\omega_{22}\left(\mathrm{ŔPC}_{\mathrm{k}-1}\right)+2$

$\omega_{23}\left(\mathrm{Ŕ}_{\mathrm{k}}^{3}\right)=\omega_{23}\left(\mathrm{ŔPC}_{\mathrm{k}-1}\right)+4$

$\omega_{33}\left(\operatorname{ŔPC}_{\mathrm{k}}^{3}\right)=\omega_{33}\left(\mathrm{Ŕ}_{\mathrm{k}-1}\right)+1$

by using Equation 5, we have:

$\mathrm{SO}_{\mathrm{avg}}\left(\mathrm{RPC}_{\mathrm{k}}^{3}\right)=\mathrm{SO}_{\mathrm{avg}}\left(\mathrm{R} P C_{\mathrm{k}-1}\right)+\left(\frac{4 \sqrt{2} \mathrm{k}-\sqrt{2}}{3 \mathrm{k}}\right)+\left(\frac{4 \sqrt{5 \mathrm{k}^{2}+2 \mathrm{k}+2}}{3 \mathrm{k}}\right)$

Thus:

$\mathrm{E}_{\mathrm{k}}^{\mathrm{avg}}=\zeta_{1} \mathrm{SO}_{\mathrm{avg}}\left(\mathrm{RPC}_{\mathrm{k}}^{1}\right)+\zeta_{2} \mathrm{SO}_{\mathrm{avg}}\left(\mathrm{R} P C_{\mathrm{k}}^{2}\right)+\left(1-\zeta_{1}-\zeta_{2}\right) \mathrm{SO}_{\mathrm{avg}}\left(\mathrm{ŔPC}_{\mathrm{k}}^{3}\right)$

$\mathrm{E}_{\mathrm{k}}^{\mathrm{avg}}=\zeta_{1} \mathrm{SO}_{\mathrm{avg}}\left(\mathrm{R}_{\mathrm{P} C} \mathrm{k-1}\right)+\left[\left(\frac{7 \sqrt{2} \mathrm{k}-\sqrt{2}}{3 \mathrm{k}}\right)+\left(\frac{2 \sqrt{5 \mathrm{k}^{2}+2 \mathrm{k}+2}}{3 \mathrm{k}}\right)\right] \zeta_{1}+\zeta_{2} \mathrm{SO}_{\text {avg }}\left(\mathrm{ŔPC}_{\mathrm{k}-1}\right)+\left[\left(\frac{4 \sqrt{2} \mathrm{k}-\sqrt{2}}{3 \mathrm{k}}\right)+\left(\frac{4 \sqrt{5 \mathrm{k}^{2}+2 \mathrm{k}+2}}{3 \mathrm{k}}\right)\right] \zeta_{2}+$

$\left(1-\zeta_{1}-\zeta_{2}\right) \mathrm{SO}_{\mathrm{avg}}\left(\mathrm{ŔPC}_{\mathrm{k}}^{3}\right)+\left(1-\zeta_{1}-\zeta_{2}\right)\left[\left(\frac{4 \sqrt{2} \mathrm{k}-\sqrt{2}}{3 \mathrm{k}}\right)+\left(\frac{4 \sqrt{5 \mathrm{k}^{2}+2 \mathrm{k}+2}}{3 \mathrm{k}}\right)\right]$

$\mathrm{E}_{\mathrm{k}}^{\mathrm{avg}}=\mathrm{SO}_{\mathrm{avg}}\left(\mathrm{RPC}_{\mathrm{k}-1}\right)+\left(\sqrt{2}-\frac{2 \sqrt{5 \mathrm{k}^{2}+2 \mathrm{k}+2}}{3 \mathrm{k}}\right) \zeta_{1}+\left[\frac{4 \sqrt{2} \mathrm{k}-\sqrt{2}}{3 \mathrm{k}}+\frac{4 \sqrt{5 \mathrm{k}^{2}+2 \mathrm{k}+2}}{3 \mathrm{k}}\right]$

Since $E\left[E_{k}\right]^{\text {avg }}=E_{k}^{a v g}$ so apply the operator $E$ on Equation 11, we get:

$\mathrm{E}_{\mathrm{k}}^{\mathrm{avg}}=\mathrm{E}_{\mathrm{k}-1}^{\mathrm{avg}}+\left[\sqrt{2}-\frac{2 \sqrt{5 \mathrm{k}^{2}+2 \mathrm{k}+2}}{3 \mathrm{k}}\right] \zeta_{1}+\left[\frac{4 \sqrt{2} \mathrm{k}-\sqrt{2}}{3 \mathrm{k}}+\frac{4 \sqrt{5 \mathrm{k}^{2}+2 \mathrm{k}+2}}{3 \mathrm{k}}\right], \mathrm{k}>2$

and after solving the recurrence relation in Equation 12 with $E_{2}^{a v g}=\frac{13 \sqrt{2}}{6}+\frac{2 \sqrt{26}}{3}$, we get:

$\mathrm{E}_{\mathrm{k}}^{\mathrm{avg}}=\left[\left[\sqrt{2}-\frac{2 \sqrt{5 \mathrm{k}^{2}+2 \mathrm{k}+2}}{3 \mathrm{k}}\right] \zeta_{1}+\left[\frac{4 \sqrt{2} \mathrm{k}-\sqrt{2}}{3 \mathrm{k}}+\frac{4 \sqrt{5 \mathrm{k}^{2}+2 \mathrm{k}+2}}{3 \mathrm{k}}\right]\right] \mathrm{k}-\left(2 \sqrt{2}-\frac{4 \sqrt{5 \mathrm{k}^{2}+2 \mathrm{k}+2}}{3 \mathrm{k}}\right) \zeta_{1}-\left(\frac{8 \sqrt{2} \mathrm{k}-2 \sqrt{2}}{3 \mathrm{k}}+\frac{8 \sqrt{5 \mathrm{k}^{2}+2 \mathrm{k}+2}}{3 \mathrm{k}}\right)+\frac{13 \sqrt{2}}{6}+\frac{2 \sqrt{26}}{3}$.

These indices can be computed for three chains meta $M_{k}=\hat{\mathrm{R}} P C(k ; 0,1)$ para $P_{k}=\hat{\mathrm{R}} P C(k ; 0,0)$ and ortho $O_{k}=$ ŔPC $(k ; 1,0)$.

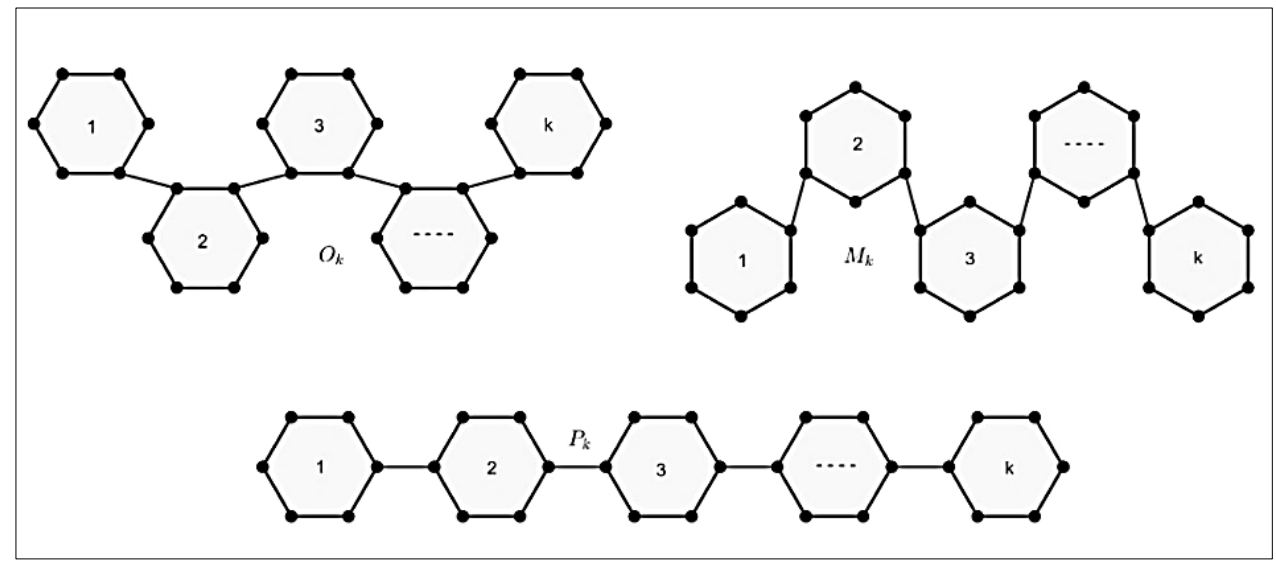

Figure 1. Polyphenyl ortho, meta and para Chains 


\section{2-4-Corollary}

For $k \geq 2$, we have:

$$
\begin{aligned}
& -\mathrm{SO}\left(\mathrm{O}_{\mathrm{k}}\right)=(12 \sqrt{2}+2 \sqrt{13}) \mathrm{k}-5 \sqrt{2} \\
& \cdot \mathrm{SO}\left(\mathrm{P}_{\mathrm{k}}\right)=\mathrm{SO}\left(\mathrm{M}_{\mathrm{k}}\right)=(7 \sqrt{2}+4 \sqrt{13}) \mathrm{k}+(5 \sqrt{2}-4 \sqrt{13}) \\
& \cdot \mathrm{SO}_{\text {red }}\left(\mathrm{O}_{\mathrm{k}}\right)=(7 \sqrt{2}+2 \sqrt{5}) \mathrm{k}-4 \sqrt{2} \\
& \cdot \mathrm{SO}_{\text {red }}\left(\mathrm{P}_{\mathrm{k}}\right)=\mathrm{SO}_{\text {red }}\left(\mathrm{M}_{\mathrm{k}}\right)=(4 \sqrt{2}+4 \sqrt{5}) \mathrm{k}+(2 \sqrt{2}-4 \sqrt{5}) \\
& -\mathrm{SO}_{\text {avg }}\left(\mathrm{O}_{\mathrm{k}}\right)=\left(\frac{7 \sqrt{2}-\sqrt{2}}{3 \mathrm{k}}+\frac{2 \sqrt{5 \mathrm{k}^{2}+2 \mathrm{k}+2}}{3 \mathrm{k}}\right) \mathrm{k}-\frac{5 \sqrt{2}}{2}+\frac{2 \sqrt{2}}{3 \mathrm{k}}-\frac{4 \sqrt{5 \mathrm{k}^{2}+2 \mathrm{k}+2}}{3 \mathrm{k}}+\frac{2 \sqrt{26}}{3} . \\
& -\mathrm{SO}_{\text {avg }}\left(\mathrm{P}_{\mathrm{k}}\right)=\mathrm{SO}_{\text {avg }}\left(\mathrm{M}_{\mathrm{k}}\right)=\left(\frac{4 \sqrt{2}-\sqrt{2}}{3 \mathrm{k}}+\frac{4 \sqrt{5 \mathrm{k}^{2}+2 \mathrm{k}+2}}{3 \mathrm{k}}\right) \mathrm{k}-\left[\frac{8 \sqrt{2 \mathrm{k}}}{3 \mathrm{k}}-\frac{2 \sqrt{2}}{3 \mathrm{k}}+\frac{8 \sqrt{5 \mathrm{k}^{2}+2 \mathrm{k}+2}}{3 \mathrm{k}}\right]+\frac{13 \sqrt{2}}{6}+\frac{2 \sqrt{26}}{3}
\end{aligned}
$$

\section{3- Average Value of Indices in Polyphenyl chains}

In this part, the average values of $\mathrm{SO}, \mathrm{SO}_{\text {red }}$ and $\mathrm{SO}_{\text {avg }}$ have been resolved for the set of random Polyphenyl chains [14]. The average values over the set $\hat{R} P_{k}$ are:

$$
\begin{aligned}
& \mathrm{SO}_{\text {avg }}\left(\mathrm{R}_{\mathrm{k}}\right)=\frac{1}{\left|\hat{\mathrm{R}} \mathrm{P}_{\mathrm{k}}\right|} \sum_{\mathrm{G} \in \hat{\mathrm{R}} \mathrm{P}_{\mathrm{k}}} \mathrm{SO}(\mathrm{G}) \\
& \left(\mathrm{SO}_{\text {red }}\right)_{\text {avg }}\left(\hat{\mathrm{R}} \mathrm{P}_{\mathrm{k}}\right)=\frac{1}{\left|\hat{\mathrm{R}} \mathrm{P}_{\mathrm{k}}\right|} \sum_{\mathrm{G} \in \hat{\mathrm{R}} \mathrm{P}_{\mathrm{k}}} \mathrm{SO}_{\text {red }}(\mathrm{G}) \\
& \left(\mathrm{SO}_{\text {avg }}\right)_{\text {avg }}\left(\hat{\mathrm{R}} \mathrm{P}_{\mathrm{k}}\right)=\frac{1}{\left|\hat{\mathrm{R}} \mathrm{P}_{\mathrm{k}}\right|} \sum_{\mathrm{G} \in \hat{\mathrm{R}} \mathrm{P}_{\mathrm{k}}} \mathrm{SO}_{\text {avg }}(\mathrm{G})
\end{aligned}
$$

As a result $\zeta_{1}=\zeta_{2}=1-\zeta_{1}-\zeta_{2}$, we can use theorem (2-1, 2-2, 2-3) by setting $\zeta_{1}=\zeta_{2}=1-\zeta_{1}-\zeta_{2}=\frac{1}{3}$ and the results will be in the form of following expression.

\section{3-1-Theorem}

Let $R P_{k}$ be the set of arbitrary Polyphenyl chains, then:

$$
\begin{aligned}
& \mathrm{SO}\left(\hat{\mathrm{R}}_{\mathrm{k}}\right)=\left[\frac{26 \sqrt{2}}{3}+\frac{10 \sqrt{13}}{3}\right] \mathrm{k}+\frac{5 \sqrt{2}}{3}-\frac{8 \sqrt{13}}{3} . \\
& \mathrm{SO}_{\text {red }}\left(\mathrm{R}_{\mathrm{k}}\right)=\left[5 \sqrt{2}+\frac{10 \sqrt{5}}{3}\right] \mathrm{k}-\frac{8 \sqrt{5}}{3} . \\
& \mathrm{SO}_{\text {avg }}\left(\mathrm{R}_{\mathrm{k}}\right)=\left[\frac{5 \sqrt{2}}{3}+\frac{\sqrt{2}}{3 \mathrm{k}}+\frac{10 \sqrt{5 \mathrm{k}^{2}+2 \mathrm{k}+2}}{9 \mathrm{k}}\right] \mathrm{k}-\frac{20 \sqrt{5 \mathrm{k}^{2}+2 \mathrm{k}+2}}{9 \mathrm{k}}-\frac{7 \sqrt{2}}{6}+\frac{2 \sqrt{2}}{3 \mathrm{k}}+\frac{2 \sqrt{26}}{3} .
\end{aligned}
$$

from corollary (2-4);

$$
\begin{aligned}
& \frac{1}{3}\left[S O\left(O_{k}\right)+S O\left(P_{k}\right)+S O\left(M_{k}\right)\right]=\left[\frac{26 \sqrt{2}}{3}+\frac{10 \sqrt{13}}{3}\right] k+\frac{5 \sqrt{2}}{3}-\frac{8 \sqrt{13}}{3} . \\
& \frac{1}{3}\left[S O\left(O_{k}\right)+S O\left(P_{k}\right)+S O\left(M_{k}\right)\right]=\left[5 \sqrt{2}+\frac{10 \sqrt{5}}{3}\right] k-\frac{8 \sqrt{5}}{3} . \\
& \frac{1}{3}\left[S O\left(O_{k}\right)+S O\left(P_{k}\right)+S O\left(M_{k}\right)\right]=\left[\frac{5 \sqrt{2}}{3}+\frac{\sqrt{2}}{3 k}+\frac{10 \sqrt{5 k^{2}+2 k+2}}{9 k}\right] k-\frac{20 \sqrt{5 k^{2}+2 k+2}}{9 k}-\frac{7 \sqrt{2}}{6}+\frac{2 \sqrt{2}}{3 k}+\frac{2 \sqrt{26}}{3} .
\end{aligned}
$$

Thus the average value of these indices $S O\left(\hat{\mathrm{R}} P C\left(k, \zeta_{1}, \zeta_{2}\right)\right), S O_{\text {red }}\left(\hat{\mathrm{R}} P C\left(k, \zeta_{1}, \zeta_{2}\right)\right)$ or $S O_{\text {avg }}\left(\hat{\mathrm{R}} P C\left(k, \zeta_{1}, \zeta_{2}\right)\right)$ is always equal over the set $\left\{O_{k}, P_{k}, M_{k}\right\}$.

\section{4- Ratio between Sombor, Reduced Sombor and Average Sombor Index for Polyphenyl Chains}

Now, by getting results from theorem $(2-1,2-2)$ and (2-3) we will assemble the ratio between the anticipated values for the $S O, S O_{\text {red }}$ and $S O_{\text {avg }}$ of an arbitrary Polyphenyl chains [15] with probability $\zeta_{1}$ and $\zeta_{2}$.

\section{4-1-Theorem}

If $k \geq 2$, then:

$E^{S O}\left[S O\left(\hat{\mathrm{R}} P C\left(k, \zeta_{1}, \zeta_{2}\right)\right)\right]>E^{\text {red }}\left[\operatorname{redSO}\left(\hat{\mathrm{R}} P C\left(k, \zeta_{1}, \zeta_{2}\right)\right)\right]$.

Proof: The statement is true for $k=2$. Thus for $k>2$, by applying theorem (2-1) and (2-2) we get; 


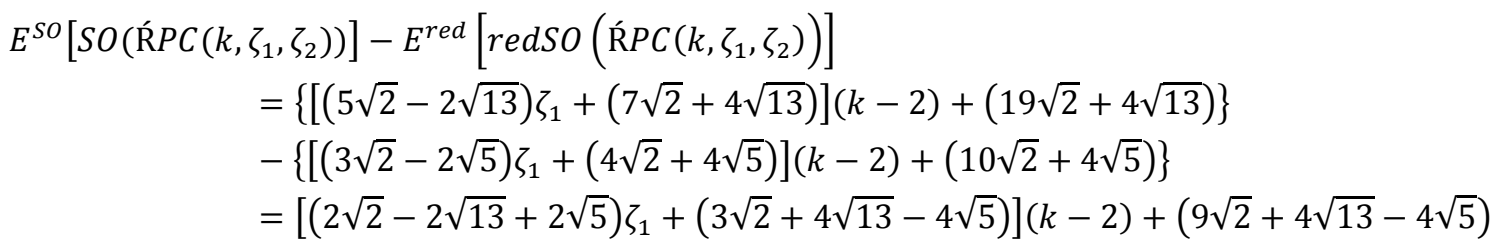

Since:

$(2 \sqrt{2}-2 \sqrt{13}+2 \sqrt{5})>0,(3 \sqrt{2}+4 \sqrt{13}-4 \sqrt{5})>0,(9 \sqrt{2}+4 \sqrt{13}-4 \sqrt{5})>0$

So

$=\left[(2 \sqrt{2}-2 \sqrt{13}+2 \sqrt{5}) \zeta_{1}+(3 \sqrt{2}+4 \sqrt{13}-4 \sqrt{5})\right](k-2)+(9 \sqrt{2}+4 \sqrt{13}-4 \sqrt{5})>0$,

$\because k \geq 2,0<\zeta_{1}<1$

\section{4-2-Theorem}

If $k \geq 2$, then:

$\mathrm{E}^{\mathrm{red}}\left[\operatorname{redSO}\left(\operatorname{R} P C\left(\mathrm{k}, \zeta_{1}, \zeta_{2}\right)\right)\right]>\mathrm{E}^{\mathrm{avg}}\left[\operatorname{avgSO}\left(\mathrm{R} P C\left(\mathrm{k}, \zeta_{1}, \zeta_{2}\right)\right)\right]$.

Proof: The statement is true for $k=2$. Thus for $k>2$, by applying theorem (2-2) and (2-3), we get;

$\mathrm{E}^{\mathrm{red}}\left[\operatorname{redSO}\left(\operatorname{R} P C\left(\mathrm{k}, \zeta_{1}, \zeta_{2}\right)\right)\right]-\mathrm{E}^{\text {avg }}\left[\operatorname{avgSO}\left(\operatorname{R} P C\left(\mathrm{k}, \zeta_{1}, \zeta_{2}\right)\right)\right]=\left\{\left[(3 \sqrt{2}-2 \sqrt{5}) \zeta_{1}+(4 \sqrt{2}+4 \sqrt{5})\right](\mathrm{k}-2)+\right.$ $(10 \sqrt{2}+4 \sqrt{5})\}-\left\{\left[\left(\sqrt{2}-\frac{2 \sqrt{5 \mathrm{k}^{2}+2 \mathrm{k}+2}}{3 \mathrm{k}}\right) \zeta_{1}+\left(\frac{4 \sqrt{2} \mathrm{k}-\sqrt{2}}{3 \mathrm{k}}+\frac{4 \sqrt{5 \mathrm{k}^{2}+2 \mathrm{k}+2}}{3 \mathrm{k}}\right)\right](\mathrm{k}-2)+\left(\frac{13 \sqrt{2}}{6}+\frac{2 \sqrt{26}}{3}\right)\right\}$

$=\left[\left(2 \sqrt{2}-2 \sqrt{5}+\frac{2 \sqrt{5 \mathrm{k}^{2}+2 \mathrm{k}+2}}{3 \mathrm{k}}\right) \zeta_{1}+\left(\frac{8 \sqrt{2}}{3}+4 \sqrt{5}-\frac{\sqrt{2}}{3 \mathrm{k}}-\frac{4 \sqrt{5 \mathrm{k}^{2}+2 \mathrm{k}+2}}{3 \mathrm{k}}\right)\right](\mathrm{k}-2)+\left(\frac{47 \sqrt{2}}{6}+4 \sqrt{5}-\frac{2 \sqrt{26}}{3}\right)$

$=\left[\left(2 \sqrt{5}-\frac{2 \sqrt{5 \mathrm{k}^{2}+2 \mathrm{k}+2}}{3 \mathrm{k}}\right)\left(2-\zeta_{1}\right)+\left(\frac{8 \sqrt{2}}{3}-\frac{\sqrt{2}}{3 \mathrm{k}}-2 \sqrt{2} \zeta_{1}\right)\right](\mathrm{k}-2)+\left(\frac{47 \sqrt{2}}{6}+4 \sqrt{5}-\frac{2 \sqrt{26}}{3}\right)$

Since:

$\left(2 \sqrt{5}-\frac{2 \sqrt{5+\frac{2}{\mathrm{k}}+\frac{2}{\mathrm{k}^{2}}}}{3}\right)>0,\left(\frac{\sqrt{2}}{3}\left(\frac{8 \mathrm{k}-1}{\mathrm{k}}\right)+2 \sqrt{2} \zeta_{1}\right)>0,\left(\frac{47 \sqrt{2}}{6}+4 \sqrt{5}-\frac{2 \sqrt{26}}{3}\right)>0, \mathrm{k} \geq 2$

So

$=\left[\left(2 \sqrt{5}-\frac{2 \sqrt{5 \mathrm{k}^{2}+2 \mathrm{k}+2}}{3 \mathrm{k}}\right)\left(2-\zeta_{1}\right)+\left(\frac{8 \sqrt{2}}{3}-\frac{\sqrt{2}}{3 \mathrm{k}}-2 \sqrt{2} \zeta_{1}\right)\right](\mathrm{k}-2)+\left(\frac{47 \sqrt{2}}{6}+4 \sqrt{5}-\frac{2 \sqrt{26}}{3}\right)>0$,

$\because \mathrm{k} \geq 2,0<\zeta_{1}<1$

\section{4-3-Theorem}

If $\mathrm{k} \geq 2$, then:

$\mathrm{E}^{\mathrm{SO}}\left[\mathrm{SO}\left(\hat{\mathrm{R} P C}\left(\mathrm{k}, \zeta_{1}, \zeta_{2}\right)\right)\right]>\mathrm{E}^{\mathrm{avg}}\left[\operatorname{avgSO}\left(\operatorname{ŔPC}\left(\mathrm{k}, \zeta_{1}, \zeta_{2}\right)\right)\right]$.

Proof: The statement is true for $\mathrm{k}=2$. Thus for $\mathrm{k}>2$, by applying theorem (2-1) and (2-3) we get;

$\mathrm{E}^{\mathrm{SO}}\left[\mathrm{SO}\left(\hat{\mathrm{R} P C}\left(\mathrm{k}, \zeta_{1}, \zeta_{2}\right)\right)\right]-\mathrm{E}^{\mathrm{avg}}\left[\operatorname{avgSO}\left(\operatorname{R} P C\left(\mathrm{k}, \zeta_{1}, \zeta_{2}\right)\right)\right]=\left\{\left[(5 \sqrt{2}-2 \sqrt{13}) \zeta_{1}+(7 \sqrt{2}+4 \sqrt{13})\right](\mathrm{k}-2)+\right.$ $(19 \sqrt{2}+4 \sqrt{13})\}-\left\{\left[\left(\sqrt{2}-\frac{2 \sqrt{5 \mathrm{k}^{2}+2 \mathrm{k}+2}}{3 \mathrm{k}}\right) \zeta_{1}+\left(\frac{4 \sqrt{2} \mathrm{k}-\sqrt{2}}{3 \mathrm{k}}+\frac{4 \sqrt{5 \mathrm{k}^{2}+2 \mathrm{k}+2}}{3 \mathrm{k}}\right)\right](\mathrm{k}-2)+\left(\frac{13 \sqrt{2}}{6}+\frac{2 \sqrt{26}}{3}\right)\right\}$

$=\left[\left(4 \sqrt{2}-2 \sqrt{13}+\frac{2 \sqrt{5 \mathrm{k}^{2}+2 \mathrm{k}+2}}{3 \mathrm{k}}\right) \zeta_{1}+\left(\frac{17 \sqrt{2}}{3}+4 \sqrt{13}-\frac{\sqrt{2}}{3 \mathrm{k}}-\frac{4 \sqrt{5 \mathrm{k}^{2}+2 \mathrm{k}+2}}{3 \mathrm{k}}\right)\right](\mathrm{k}-2)+\left(\frac{101 \sqrt{2}}{6}+4 \sqrt{13}-\frac{2 \sqrt{26}}{3}\right)$

$=\left[\left(2 \sqrt{13}-\frac{2 \sqrt{5 \mathrm{k}^{2}+2 \mathrm{k}+2}}{3 \mathrm{k}}\right)\left(2-\zeta_{1}\right)+\left(\frac{17 \sqrt{2}}{3}-\frac{\sqrt{2}}{3 \mathrm{k}}-4 \sqrt{2} \zeta_{1}\right)\right](\mathrm{k}-2)+\left(\frac{101 \sqrt{2}}{6}+4 \sqrt{13}-\frac{2 \sqrt{26}}{3}\right)$

Since

$\left(2 \sqrt{13}-\frac{2 \sqrt{5+\frac{2}{\mathrm{k}^{+}}+\frac{2}{\mathrm{k}^{2}}}}{3}\right)>0,\left(\frac{101 \sqrt{2}}{6}+4 \sqrt{13}-\frac{2 \sqrt{26}}{3}\right)>0,\left(\frac{8 \sqrt{2}}{3}-\frac{\sqrt{2}}{3 \mathrm{k}}+2 \sqrt{2} \zeta_{1}\right)>0, \mathrm{k} \geq 2$. 
So

$=\left[\left(2 \sqrt{13}-\frac{2 \sqrt{5 \mathrm{k}^{2}+2 \mathrm{k}+2}}{3 \mathrm{k}}\right)\left(2-\zeta_{1}\right)+\left(\frac{17 \sqrt{2}}{3}-\frac{\sqrt{2}}{3 \mathrm{k}}-4 \sqrt{2} \zeta_{1}\right)\right](\mathrm{k}-2)+\left(\frac{101 \sqrt{2}}{6}+4 \sqrt{13}-\frac{2 \sqrt{26}}{3}\right)>0$,

$\because \mathrm{k} \geq 2,0<\zeta_{1}<1$.

\section{4-4-Corollary}

For $k \geq 2, E^{S O}\left[S O\left(\hat{R} P C\left(k, \zeta_{1}, \zeta_{2}\right)\right)\right]>E^{r e d}\left[\operatorname{redSO}\left(\hat{\mathrm{R}} P C\left(k, \zeta_{1}, \zeta_{2}\right)\right)\right]>E^{\operatorname{avg}}\left[\operatorname{avgSO}\left(\hat{\mathrm{R}} P C\left(k, \zeta_{1}, \zeta_{2}\right)\right)\right]$ as shown in Figure 4.

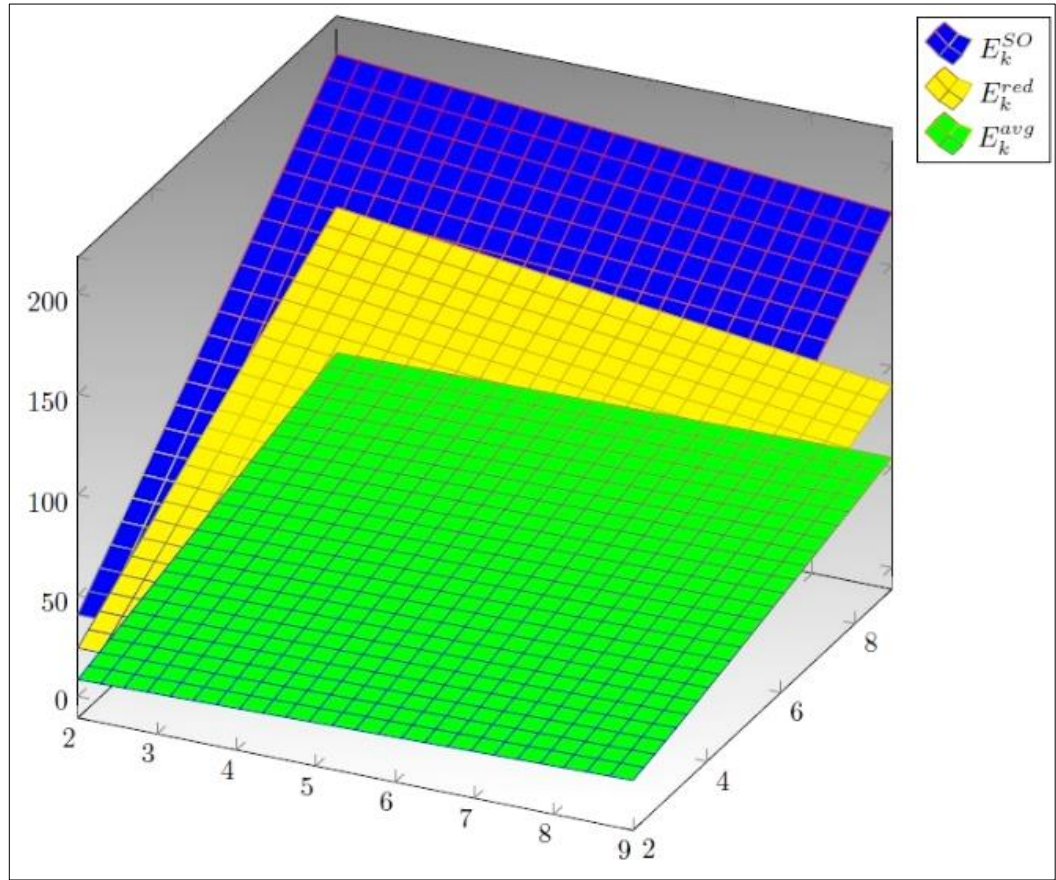

Figure 2. Comparison between the expected values of Sombor $E_{k}^{S O}$ (blue), reduced Sombor $E_{k}^{\text {red }}$ (yellow) and average Sombor $E_{k}^{a v g}$ (green) index in random Polyphenyl chains

\section{5- Sombor, Reduced Sombor and Average Sombor Index in Random Spiro Chain}

In the following part, we consider the $S O, S O_{\text {red }}$ and $S O_{a v g}$ in the arbitrary spiro-chain $R S C_{k}$. Let $R S C_{k}$ be the spiro chain [16-20] acquired by ŔSC $C_{k-1}$. Clearly, there are only $(2,2),(2,4)$ and $(4,4)-$ edges in Ŕ $S C_{k}$. By using the definition of $\mathrm{SO}, \mathrm{SO}_{\text {red }}$ and $\mathrm{SO}_{\mathrm{avg}}$, we have the following equation:

$$
\begin{aligned}
& \mathrm{SO}\left(\mathrm{RSC}_{\mathrm{k}}\right)=2 \sqrt{2} \omega_{22}\left(\mathrm{ŔSC}_{\mathrm{k}}\right)+2 \sqrt{5} \omega_{24}\left(\mathrm{ŔSC}_{\mathrm{k}}\right)+4 \sqrt{2} \omega_{44}\left(\mathrm{ŔSC}_{\mathrm{k}}\right) \\
& \mathrm{SO}_{\text {red }}\left(\mathrm{ŔSC}_{\mathrm{k}}\right)=\sqrt{2} \omega_{22}\left(\mathrm{ŔSC}_{\mathrm{k}}\right)+\sqrt{10} \omega_{24}\left(\mathrm{ŔSC}_{\mathrm{k}}\right)+3 \sqrt{2} \omega_{44}\left(\mathrm{ŔSC}_{\mathrm{k}}\right) \\
& \mathrm{SO}_{\mathrm{avg}}\left(\operatorname{RSCC}_{\mathrm{k}}\right)=2 \sqrt{2}\left(\frac{\mathrm{k}-1}{5 \mathrm{k}+1}\right) \omega_{22}\left(\operatorname{RSC}_{\mathrm{k}}\right)+\frac{2 \sqrt{17 \mathrm{k}^{2}+14 \mathrm{k}+5}}{5 \mathrm{k}+1} \omega_{24}\left(\operatorname{ŔSC}_{\mathrm{k}}\right)+4 \sqrt{2}\left(\frac{2 \mathrm{k}+1}{5 \mathrm{k}+1}\right) \omega_{44}\left(\operatorname{ŔSC}_{\mathrm{k}}\right)
\end{aligned}
$$

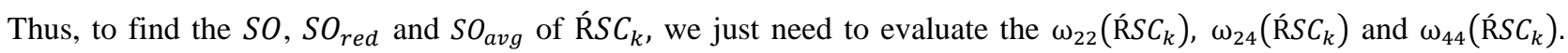
Since ŔSC $\left(k ; \zeta_{1}, \zeta_{2}\right)$ is an arbitrary spiro-chain. So, $S O\left(\hat{\mathrm{R} S C}\left(k ; \zeta_{1}, \zeta_{2}\right)\right), S O_{\text {red }}\left(\hat{\mathrm{R} S C}\left(k ; \zeta_{1}, \zeta_{2}\right)\right)$ and $S O_{\text {avg }}\left(\hat{\mathrm{R}} S C\left(k ; \zeta_{1}, \zeta_{2}\right)\right)$ are also arbitrary variables. We symbolize the anticipated values of these indices by $E_{k}^{S O}=$ $E^{S O}\left[S O\left(\hat{R} S C\left(k ; \zeta_{1}, \zeta_{2}\right)\right)\right], E_{k}^{r e d}=E^{\text {red }}\left[S O_{\text {red }}\left(\hat{\mathrm{R}} S C\left(k ; \zeta_{1}, \zeta_{2}\right)\right)\right]$ and $E_{k}^{\text {avg }}=E^{\text {avg }}\left[S O_{\text {avg }}\left(\hat{\mathrm{R}} S C\left(k ; \zeta_{1}, \zeta_{2}\right)\right)\right]$ respectively.

\section{5-1-Theorem}

Let ŔSC $\left(k ; \zeta_{1}, \zeta_{2}\right)$ be an arbitrary spiro-chain of length $k$, where $k \geq 2$. Then: $\mathrm{E}_{\mathrm{k}}^{\mathrm{SO}}=\left[(6 \sqrt{2}-4 \sqrt{5}) \zeta_{1}+(4 \sqrt{2}+8 \sqrt{5})\right] \mathrm{k}-(12 \sqrt{2}-8 \sqrt{5}) \zeta_{1}+(8 \sqrt{2}-8 \sqrt{5})$.

Proof: For $k=2$, we have $E_{2}^{S O}=16 \sqrt{2}+8 \sqrt{5}$. Now, when $k \geq 3$, it is obvious that $\omega_{22}, \omega_{24}$ and $\omega_{44}$ depend on the following three cases: 
1. If Ŕ $S C_{k-1} \rightarrow R ́ R C_{k}^{1}$ with probability $\zeta_{1}$

$\omega_{22}\left(\hat{R} S C_{k}^{1}\right)=\omega_{22}\left(\hat{R} S C_{k-1}\right)+3$

$\omega_{24}\left(\hat{R} S C_{k}^{1}\right)=\omega_{24}\left(\hat{R} S C_{k-1}\right)+2$

$\omega_{44}\left(\hat{R} S C_{k}^{1}\right)=\omega_{44}\left(\hat{R} S C_{k-1}\right)+1$

by using Equation 13:

$\mathrm{SO}\left(\operatorname{ŔSC}_{\mathrm{k}}^{1}\right)=\mathrm{SO}\left(\mathrm{ŔSC}_{\mathrm{k}-1}\right)+10 \sqrt{2}+4 \sqrt{5}$

2. If Ŕ $S C_{k-1} \rightarrow R ́ R C_{k}^{2}$ with probability $\zeta_{2}$

$\omega_{22}\left(\hat{\mathrm{R}} S C_{k}^{2}\right)=\omega_{22}\left(\hat{\mathrm{R}} S C_{k-1}\right)+2$

$\omega_{24}\left(\hat{\mathrm{R}} S C_{k}^{2}\right)=\omega_{24}\left(\hat{\mathrm{R}} S C_{k-1}\right)+4$

$\omega_{44}\left(\hat{\mathrm{R} S} C_{k}^{2}\right)=\omega_{44}\left(\hat{\mathrm{R} S} C_{k-1}\right)$

by using Equation 13:

$\mathrm{SO}\left(\mathrm{ŔSC}_{\mathrm{k}}^{2}\right)=\mathrm{SO}\left(\mathrm{ŔSC}_{\mathrm{k}-1}\right)+4 \sqrt{2}+8 \sqrt{5}$

3. If Ŕ $S C_{k-1} \rightarrow$ ŔSC $C_{k}^{3}$ with probability $1-\zeta_{1}-\zeta_{2}$

$\omega_{22}\left(\hat{\mathrm{R} S} C_{k}^{3}\right)=\omega_{22}\left(\hat{\mathrm{R}} S C_{k-1}\right)+2$

$\omega_{24}\left(\hat{R} S C_{k}^{3}\right)=\omega_{24}\left(\hat{R} S C_{k-1}\right)+4$

$\omega_{44}\left(\hat{R} S C_{k}^{3}\right)=\omega_{44}\left({\left.\operatorname{Ŕ} S C_{k-1}\right)}\right)$

by using Equation 13:

$\mathrm{SO}\left(\mathrm{ŔSC}_{\mathrm{k}}^{3}\right)=\mathrm{SO}\left(\mathrm{ŔSC}_{\mathrm{k}-1}\right)+4 \sqrt{2}+8 \sqrt{5}$

Thus:

$\mathrm{E}_{\mathrm{k}}^{\mathrm{SO}}=\zeta_{1} \mathrm{SO}\left(\mathrm{ŔSC}_{\mathrm{k}}^{1}\right)+\zeta_{2} \mathrm{SO}\left(\mathrm{ŔSC}_{\mathrm{k}}^{2}\right)+\left(1-\zeta_{1}-\zeta_{2}\right) \mathrm{SO}\left(\mathrm{ŔSC}_{\mathrm{k}}^{3}\right)$

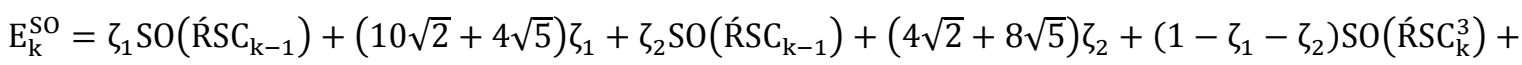
$\left(1-\zeta_{1}-\zeta_{2}\right)(4 \sqrt{2}+8 \sqrt{5})$

$\mathrm{E}_{\mathrm{k}}^{\mathrm{SO}}=\mathrm{SO}\left(\mathrm{ŔSC}_{\mathrm{k}-1}\right)+(6 \sqrt{2}-4 \sqrt{5}) \zeta_{1}+(4 \sqrt{2}+8 \sqrt{5})$

Since $E\left[E_{k}\right]^{S O}=E_{k}^{S O}$ so apply the operator $E$ on Equation 16 , we get:

$\mathrm{E}_{\mathrm{k}}^{\mathrm{SO}}=\mathrm{E}_{\mathrm{k}-1}^{\mathrm{SO}}+(6 \sqrt{2}-4 \sqrt{5}) \zeta_{1}+(4 \sqrt{2}+8 \sqrt{5}), \quad \mathrm{k}>2$

and after solving the recurrence relation in Equation 17 with $E_{2}^{S O}=16 \sqrt{2}+8 \sqrt{5}$, we get:

$\mathrm{E}_{\mathrm{k}}^{\mathrm{SO}}=\left[(6 \sqrt{2}-4 \sqrt{5}) \zeta_{1}+(4 \sqrt{2}+8 \sqrt{5})\right] \mathrm{k}-(12 \sqrt{2}-8 \sqrt{5}) \zeta_{1}+(8 \sqrt{2}-8 \sqrt{5})$

\section{5-2-Theorem}

Let ŔSC $\left(k ; \zeta_{1}, \zeta_{2}\right)$ be an arbitrary spiro chain of length $k$, where $k \geq 2$. Then;

$\mathrm{E}_{\mathrm{k}}^{\mathrm{red}}=\left[(4 \sqrt{2}-2 \sqrt{10}) \zeta_{1}+(2 \sqrt{2}+4 \sqrt{10})\right] \mathrm{k}-(8 \sqrt{2}-4 \sqrt{10}) \zeta_{1}+(4 \sqrt{2}-4 \sqrt{10})$.

Proof: For $k=2$, we have $E_{2}^{\text {red }}=8 \sqrt{2}+4 \sqrt{10}$. Now, when $k \geq 3$, it is obvious that $\omega_{22}, \omega_{24}$ and $\omega_{44}$ depend on the following three cases:

1. If Ŕ $S C_{k-1} \rightarrow R ́ R C_{k}^{1}$ with probability $\zeta_{1}$

$\omega_{22}\left(\hat{R} S C_{k}^{1}\right)=\omega_{22}\left(\hat{R} S C_{k-1}\right)+3$

$\omega_{24}\left(\hat{R} S C_{k}^{1}\right)=\omega_{24}\left(\hat{R} S C_{k-1}\right)+2$

$\omega_{44}\left(\hat{R} S C_{k}^{1}\right)=\omega_{44}\left(\hat{R} S C_{k-1}\right)+1$

by using Equation 14: 
$\mathrm{SO}_{\text {red }}\left(\mathrm{ŔSC}_{\mathrm{k}}^{1}\right)=\mathrm{SO}_{\text {red }}\left(\mathrm{ŔSC}_{\mathrm{k}-1}\right)+6 \sqrt{2}+2 \sqrt{10}$

2. If $\mathrm{R} S C_{k-1} \rightarrow \mathrm{R} S C_{k}^{2}$ with probability $\zeta_{2}$

$\omega_{22}\left(\hat{R} S C_{k}^{2}\right)=\omega_{22}\left(\hat{R} S C_{k-1}\right)+2$

$\omega_{24}\left(\hat{R} S C_{k}^{2}\right)=\omega_{24}\left(\hat{R} S C_{k-1}\right)+4$

$\omega_{44}\left(\hat{R} S C_{k}^{2}\right)=\omega_{44}\left(\hat{R ́} C_{k-1}\right)$

by using Equation 14:

$\mathrm{SO}_{\text {red }}\left(\mathrm{ŔSC}_{\mathrm{k}}^{2}\right)=\mathrm{SO}_{\text {red }}\left(\mathrm{ŔSC}_{\mathrm{k}-1}\right)+2 \sqrt{2}+4 \sqrt{10}$

3. If Ŕ $S C_{k-1} \rightarrow$ ŔSC$C_{k}^{3}$ with probability $1-\zeta_{1}-\zeta_{2}$

$\omega_{22}\left(\hat{R} S C_{k}^{3}\right)=\omega_{22}\left(\hat{R}_{S} C_{k-1}\right)+2$

$\omega_{24}\left(\hat{R} S C_{k}^{3}\right)=\omega_{24}\left(\hat{R} S C_{k-1}\right)+4$

$\omega_{44}\left(\hat{R} S C_{k}^{3}\right)=\omega_{44}\left(\hat{R} S C_{k-1}\right)$

by using Equation 14:

$\mathrm{SO}_{\text {red }}\left(\mathrm{ŔSC}_{\mathrm{k}}^{3}\right)=\mathrm{SO}_{\text {red }}\left(\mathrm{ŔSC}_{\mathrm{k}-1}\right)+2 \sqrt{2}+4 \sqrt{10}$

Thus:

$\mathrm{E}_{\mathrm{k}}^{\mathrm{red}}=\zeta_{1} \mathrm{SO}_{\text {red }}\left(\mathrm{RSCC}_{\mathrm{k}}^{1}\right)+\zeta_{2} \mathrm{SO}_{\text {red }}\left(\mathrm{RSCC}_{\mathrm{k}}^{2}\right)+\left(1-\zeta_{1}-\zeta_{2}\right) \mathrm{SO}_{\text {red }}\left(\mathrm{ŔSC}_{\mathrm{k}}^{3}\right)$

$\mathrm{E}_{\mathrm{k}}^{\mathrm{red}}=\zeta_{1} \mathrm{SO}_{\text {red }}\left(\mathrm{RSSC}_{\mathrm{k}-1}\right)+(6 \sqrt{2}+2 \sqrt{10}) \zeta_{1}+\zeta_{2} \mathrm{SO}_{\text {red }}\left(\mathrm{ŔSC}_{\mathrm{k}-1}\right)+(2 \sqrt{2}+4 \sqrt{10}) \zeta_{2}+\left(1-\zeta_{1}-\right.$

$\left.\zeta_{2}\right) \mathrm{SO}_{\text {red }}\left(\mathrm{RSC}_{\mathrm{k}}^{3}\right)+\left(1-\zeta_{1}-\zeta_{2}\right)(2 \sqrt{2}+4 \sqrt{10})$

$\mathrm{E}_{\mathrm{k}}^{\mathrm{red}}=\mathrm{SO}_{\mathrm{red}}\left(\mathrm{ŔSC}_{\mathrm{k}-1}\right)+(4 \sqrt{2}-2 \sqrt{10}) \zeta_{1}+(2 \sqrt{2}+4 \sqrt{10})$

Since $E\left[E_{k}\right]^{\text {red }}=E_{k}^{r e d}$ so apply the operator $E$ on Equation 18, we get;

$\mathrm{E}_{\mathrm{k}}^{\mathrm{red}}=\mathrm{E}_{\mathrm{k}-1}^{\mathrm{red}}+(4 \sqrt{2}-2 \sqrt{10}) \zeta_{1}+(2 \sqrt{2}+4 \sqrt{10}), \quad \mathrm{k}>2$

and after solving the recurrence relation in Equation 19 with initial condition, we get:

$\mathrm{E}_{\mathrm{k}}^{\mathrm{red}}=\left[(4 \sqrt{2}-2 \sqrt{10}) \zeta_{1}+(2 \sqrt{2}+4 \sqrt{10})\right] \mathrm{k}-(8 \sqrt{2}-4 \sqrt{10}) \zeta_{1}+(4 \sqrt{2}-4 \sqrt{10})$

\section{5-3-Theorem}

Let $R S C\left(k ; \zeta_{1}, \zeta_{2}\right)$ be an arbitrary spiro chain of length $k$, where $k \geq 2$. Then;

$\mathrm{E}_{\mathrm{k}}^{\mathrm{avg}}=\left[\left(\frac{10 \sqrt{2} \mathrm{k}+2 \sqrt{2}}{5 \mathrm{k}+1}-\frac{4 \sqrt{17 \mathrm{k}^{2}+14 \mathrm{k}+5}}{5 \mathrm{k}+1}\right) \zeta_{1}+\left(\frac{4 \sqrt{2} \mathrm{k}-4 \sqrt{2}}{5 \mathrm{k}+1}+\frac{8 \sqrt{17 \mathrm{k}^{2}+14 \mathrm{k}+5}}{5 \mathrm{k}+1}\right)\right] \mathrm{k}-\left(\frac{20 \sqrt{2} \mathrm{k}+4 \sqrt{2}}{5 \mathrm{k}+1}-\frac{8 \sqrt{17 \mathrm{k}^{2}+14 \mathrm{k}+5}}{5 \mathrm{k}+1}\right) \zeta_{1}+$ $\left(\frac{8 \sqrt{2} \mathrm{k}-4 \sqrt{2}}{5 \mathrm{k}+1}+\frac{16 \sqrt{17 \mathrm{k}^{2}+14 \mathrm{k}+5}}{5 \mathrm{k}+1}\right)+\frac{16 \sqrt{2}}{11}+\frac{8 \sqrt{101}}{11}$.

Proof: For $k=2$, we have $E_{2}^{a v g}=\frac{16 \sqrt{2}}{11}+\frac{8 \sqrt{101}}{11}$. Now, when $k \geq 3$, it is obvious that $\omega_{22}, \omega_{24}$ and $\omega_{44}$ depend on the following three cases:

1. If Ŕ $S C_{k-1} \rightarrow \mathrm{R} S C_{k}^{1}$ with probability $\zeta_{1}$

$\omega_{22}\left(\hat{R} S C_{k}^{1}\right)=\omega_{22}\left(\hat{R} S C_{k-1}\right)+3$

$\omega_{24}\left(\hat{R} S C_{k}^{1}\right)=\omega_{24}\left(\hat{R} S C_{k-1}\right)+2$

$\omega_{44}\left(\hat{R} S C_{k}^{1}\right)=\omega_{44}\left(\hat{R} S C_{k-1}\right)+1$

by using Equation 15:

$\mathrm{SO}_{\text {avg }}\left(\operatorname{ŔSC}_{\mathrm{k}}^{1}\right)=\mathrm{SO}_{\mathrm{avg}}\left(\operatorname{ŔSC}_{\mathrm{k}-1}\right)+\left(\frac{14 \sqrt{2} \mathrm{k}-2 \sqrt{2}}{5 \mathrm{k}+1}\right)+\left(\frac{4 \sqrt{17 \mathrm{k}^{2}+14 \mathrm{k}+5}}{5 \mathrm{k}+1}\right)$

2. If $\hat{\mathrm{R}} S C_{k-1} \rightarrow \mathrm{R} S C_{k}^{2}$ with probability $\zeta_{2}$ 
$\omega_{22}\left(\hat{R} S C_{k}^{2}\right)=\omega_{22}\left(\hat{R} S C_{k-1}\right)+2$

$\omega_{24}\left(\hat{R} S C_{k}^{2}\right)=\omega_{24}\left(\hat{R} S C_{k-1}\right)+4$

$\omega_{44}\left(\hat{\mathrm{R} S} C_{k}^{2}\right)=\omega_{44}\left(\hat{\mathrm{R}} S C_{k-1}\right)$

by using Equation 15:

$S O_{\text {avg }}\left(\hat{\mathrm{R} S} C_{k}^{2}\right)=S O_{\text {avg }}\left(\hat{\mathrm{R} S} C_{k-1}\right)+4 \sqrt{2}\left(\frac{k-1}{5 k+1}\right)+\left(\frac{8 \sqrt{17 k^{2}+14 k+5}}{5 k+1}\right)$

3. If $\mathrm{R} S C_{k-1} \rightarrow \mathrm{R} S C_{k}^{3}$ with probability $1-\zeta_{1}-\zeta_{2}$

$\omega_{22}\left(\operatorname{ŔSC}_{\mathrm{k}}^{3}\right)=\omega_{22}\left(\mathrm{ŔSC}_{\mathrm{k}-1}\right)+2$

$\omega_{24}\left(\hat{R S C}_{\mathrm{k}}^{3}\right)=\omega_{24}\left(\mathrm{RSC}_{\mathrm{k}-1}\right)+4$

$\omega_{44}\left(\operatorname{ŔSC}_{\mathrm{k}}^{3}\right)=\omega_{44}\left(\right.$ ŔSC $\left._{\mathrm{k}-1}\right)$

by using Equation 15:

$S O_{\text {avg }}\left(\hat{\mathrm{R} S} C_{k}^{3}\right)=S O_{\text {avg }}\left(\hat{\mathrm{R} S} C_{k-1}\right)+4 \sqrt{2}\left(\frac{k-1}{5 k+1}\right)+\left(\frac{8 \sqrt{17 k^{2}+14 k+5}}{5 k+1}\right)$

Thus:

$\mathrm{E}_{\mathrm{k}}^{\text {avg }}=\zeta_{1} \mathrm{SO}_{\mathrm{avg}}\left(\mathrm{RSC}_{\mathrm{k}}^{1}\right)+\zeta_{2} \mathrm{SO}_{\mathrm{avg}}\left(\mathrm{RSCC}_{\mathrm{k}}^{2}\right)+\left(1-\zeta_{1}-\zeta_{2}\right) \mathrm{SO}_{\mathrm{avg}}\left(\mathrm{ŔSC}_{\mathrm{k}}^{3}\right)$

$\mathrm{E}_{\mathrm{k}}^{\mathrm{avg}}=\zeta_{1} \mathrm{SO}_{\mathrm{avg}}\left(\mathrm{R} S C_{\mathrm{k}-1}\right)+\left[\left(\frac{14 \sqrt{2} \mathrm{k}-2 \sqrt{2}}{5 \mathrm{k}+1}\right)+\left(\frac{4 \sqrt{17 \mathrm{k}^{2}+14 \mathrm{k}+5}}{5 \mathrm{k}+1}\right)\right] \zeta_{1}+\zeta_{2} \mathrm{SO}_{\mathrm{avg}}\left(\mathrm{R} S C_{\mathrm{k}-1}\right)+\left[\left(\frac{4 \sqrt{2} \mathrm{k}-4 \sqrt{2}}{5 \mathrm{k}+1}\right)+\right.$

$\left.\left(\frac{8 \sqrt{17 \mathrm{k}^{2}+14 \mathrm{k}+5}}{5 \mathrm{k}+1}\right)\right] \zeta_{2}+\left(1-\zeta_{1}-\zeta_{2}\right) \mathrm{SO}_{\mathrm{avg}}\left(\mathrm{RSC}_{\mathrm{k}}^{3}\right)+\left(1-\zeta_{1}-\zeta_{2}\right)\left[\left(\frac{4 \sqrt{2} \mathrm{k}-4 \sqrt{2}}{5 \mathrm{k}+1}\right)+\left(\frac{8 \sqrt{17 \mathrm{k}^{2}+14 \mathrm{k}+5}}{5 \mathrm{k}+1}\right)\right]$

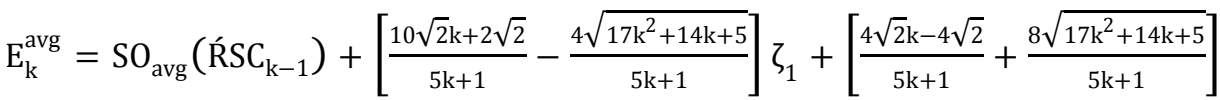

Since $E\left[E_{k}\right]^{a v g}=E_{k}^{a v g}$ so apply the operator $E$ on Equation 20, we get:

$\mathrm{E}_{\mathrm{k}}^{\text {avg }}=\mathrm{E}_{\mathrm{k}-1}^{\mathrm{avg}}+\left[\frac{10 \sqrt{2} \mathrm{k}+2 \sqrt{2}}{5 \mathrm{k}+1}-\frac{4 \sqrt{17 \mathrm{k}^{2}+14 \mathrm{k}+5}}{5 \mathrm{k}+1}\right] \zeta_{1}+\left[\frac{4 \sqrt{2} \mathrm{k}-4 \sqrt{2}}{5 \mathrm{k}+1}+\frac{8 \sqrt{17 \mathrm{k}^{2}+14 \mathrm{k}+5}}{5 \mathrm{k}+1}\right], \quad \mathrm{k}>2$

And after solving the recurrence relation in Equation 21 with initial condition, we get:

$$
\begin{aligned}
& \mathrm{E}_{\mathrm{k}}^{\mathrm{avg}}=\left[\left(\frac{10 \sqrt{2} \mathrm{k}+2 \sqrt{2}}{5 \mathrm{k}+1}-\frac{4 \sqrt{17 \mathrm{k}^{2}+14 \mathrm{k}+5}}{5 \mathrm{k}+1}\right) \zeta_{1}+\left(\frac{10 \sqrt{2} \mathrm{k}+2 \sqrt{2}}{5 \mathrm{k}+1}-\frac{4 \sqrt{17 \mathrm{k}^{2}+14 \mathrm{k}+5}}{5 \mathrm{k}+1}\right)\right] \mathrm{k}-\left(\frac{20 \sqrt{2} \mathrm{k}+4 \sqrt{2}}{5 \mathrm{k}+1}-\frac{8 \sqrt{17 \mathrm{k}^{2}+14 \mathrm{k}+5}}{5 \mathrm{k}+1}\right) \zeta_{1}- \\
& \left(\frac{8 \sqrt{2} \mathrm{k}-8 \sqrt{2}}{5 \mathrm{k}+1}+\frac{16 \sqrt{17 \mathrm{k}^{2}+14 \mathrm{k}+5}}{5 \mathrm{k}+1}\right)+\frac{16 \sqrt{2}}{11}+\frac{8 \sqrt{101}}{11} .
\end{aligned}
$$

These indices can be computed for three chains meta $\overline{M_{k}}=\operatorname{R} S C(k ; 0,1)$, para $\overline{P_{k}}=\operatorname{R} S C(k ; 0,0)$ and ortho $\overline{O_{k}}=$ ŔSC $(k ; 1,0)$ as shown in Figure 5.

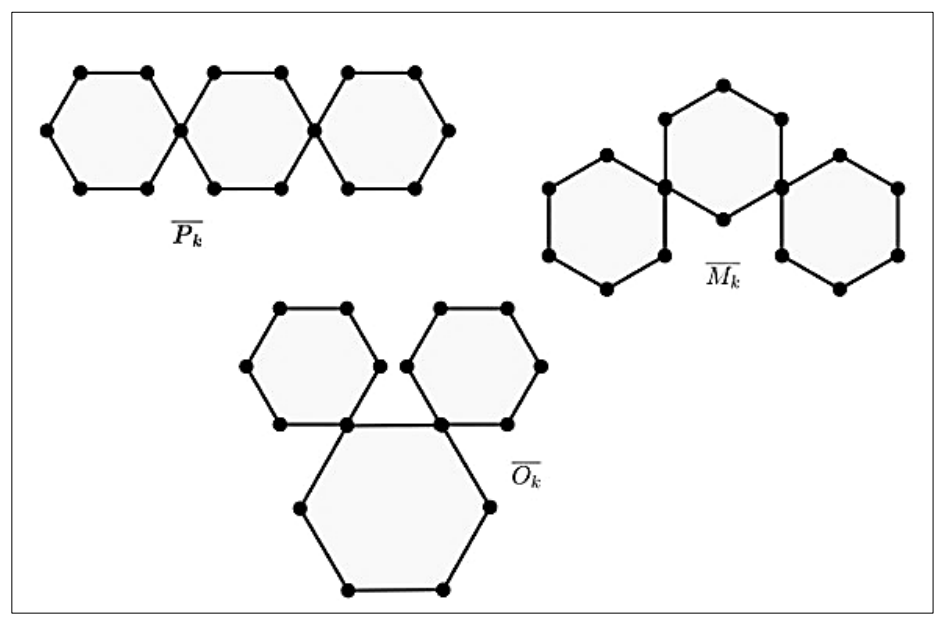

Figure 5. Special spiro ortho, meta and para chains 


\section{5-4-Corollary}

For $k \geq 2$, we have:

$$
\begin{aligned}
& \cdot S O\left(\overline{O_{k}}\right)=(10 \sqrt{2}+4 \sqrt{5}) k-4 \sqrt{2} \\
& \cdot S O\left(\overline{P_{k}}\right)=S O\left(\overline{M_{k}}\right)=(4 \sqrt{2}+8 \sqrt{5}) k+(8 \sqrt{2}-8 \sqrt{5}) \\
& \cdot S O_{\text {red }}\left(\overline{O_{k}}\right)=(6 \sqrt{2}+2 \sqrt{10}) k-4 \sqrt{2} \\
& \cdot S O_{\text {red }}\left(\overline{P_{k}}\right)=S O_{\text {red }}\left(\overline{M_{k}}\right)=(2 \sqrt{2}+4 \sqrt{10}) k+(4 \sqrt{2}-4 \sqrt{10}) \\
& -S O_{\text {avg }}\left(\overline{O_{k}}\right)=\left(\frac{14 \sqrt{2} k+4 \sqrt{2}}{5 k+1}+\frac{4 \sqrt{17 k^{2}+14 k+5}}{5 k+1}\right) k+\frac{28 \sqrt{2} k}{5 k+1}+\frac{4 \sqrt{2}}{5 k+1}-\frac{8 \sqrt{17 k^{2}+14 k+5}}{5 k+1}+\frac{16 \sqrt{2}}{11}+\frac{8 \sqrt{101}}{11} \\
& -S O_{\text {avg }}\left(\overline{P_{k}}\right)=S O_{\text {avg }}\left(\overline{M_{k}}\right)=\left(\frac{4 \sqrt{2} k-4 \sqrt{2}}{5 k+1}+\frac{8 \sqrt{17 k^{2}+14 k+5}}{5 k+1}\right) k+\frac{28 \sqrt{2} k}{5 k+1}+\frac{4 \sqrt{2}}{5 k+1}-\frac{8 \sqrt{17 k^{2}+14 k+5}}{5 k+1}+\frac{16 \sqrt{2}}{11}+\frac{8 \sqrt{101}}{11}
\end{aligned}
$$

\section{6- Average Value of Indices for Spiro Chain}

In this part, the average value of $S O, S O_{\text {red }}$ and $\mathrm{SO}_{\text {avg }}$ have been resolved for the set of arbitrary spiro chain. The average values over the set $S P_{k}$ are:

$$
\begin{aligned}
& \mathrm{SO}_{\text {avg }}\left(\mathrm{SP}_{\mathrm{k}}\right)=\frac{1}{\left|\mathrm{SP}_{\mathrm{k}}\right|} \sum_{\mathrm{G} \in \mathrm{SP}_{\mathrm{k}}} \mathrm{SO}(\mathrm{G}) \\
& \left(\mathrm{SO}_{\text {red }}\right)_{\mathrm{avg}}\left(\mathrm{SP}_{\mathrm{k}}\right)=\frac{1}{\left|\mathrm{SP}_{\mathrm{k}}\right|} \sum_{\mathrm{G} \in \mathrm{SP}_{\mathrm{k}}} \mathrm{SO}_{\text {red }}(\mathrm{G}) \\
& \left(\mathrm{SO}_{\text {avg }}\right)_{\mathrm{avg}}\left(\mathrm{SP}_{\mathrm{k}}\right)=\frac{1}{\left|\mathrm{SP}_{\mathrm{k}}\right|} \sum_{\mathrm{G} \in \mathrm{SP}_{\mathrm{k}}} \mathrm{SO}_{\text {avg }}(\mathrm{G})
\end{aligned}
$$

As a result $\zeta_{1}=\zeta_{2}=1-\zeta_{1}-\zeta_{2}$, we can use theorems (5-1, 5-2, 5-3) by setting $\zeta_{1}=\zeta_{2}=1-\zeta_{1}-\zeta_{2}=\frac{1}{3}$ and the results will be in the form of following expression:

\section{6-1-Theorem}

Let $S P_{k}$ be the set of spiro chain, then;

$$
\begin{aligned}
& \mathrm{SO}\left(\mathrm{R}_{\mathrm{k}}\right)=\left[16 \sqrt{2}+\frac{20 \sqrt{5}}{3}\right] \mathrm{k}+4 \sqrt{2}-\frac{16 \sqrt{5}}{3} . \\
& \mathrm{SO}_{\text {red }}\left(\mathrm{R}_{\mathrm{k}}\right)=\left[\frac{10 \sqrt{2}}{3}+\frac{10 \sqrt{10}}{3}\right] \mathrm{k}+\frac{4 \sqrt{2}}{3}-\frac{8 \sqrt{10}}{3} . \\
& \mathrm{SO}_{\text {avg }}\left(\mathrm{R}_{\mathrm{k}}\right)=\left[\frac{22 \sqrt{2} \mathrm{k}}{3(5 \mathrm{k}+1)}-\frac{10 \sqrt{2}}{3(5 \mathrm{k}+1)}+\frac{20 \sqrt{17 \mathrm{k}^{2}+14 \mathrm{k}+5}}{3(5 \mathrm{k}+1)}\right] \mathrm{k}-\frac{44 \sqrt{2}}{3(5 \mathrm{k}+1)}+\frac{20 \sqrt{2}}{3(5 \mathrm{k}+1)}-\frac{40 \sqrt{17 \mathrm{k}^{2}+14 \mathrm{k}+5}}{3(5 \mathrm{k}+1)}+\frac{16 \sqrt{2}}{11}+\frac{8 \sqrt{101}}{11} .
\end{aligned}
$$

from corollary (5-4);

$$
\begin{aligned}
& \frac{1}{3}\left[S O\left(\overline{O_{k}}\right)+S O\left(\overline{P_{k}}\right)+S O\left(\overline{M_{k}}\right)\right]=\left[16 \sqrt{2}+\frac{20 \sqrt{5}}{3}\right] k+4 \sqrt{2}-\frac{16 \sqrt{5}}{3} . \\
& \frac{1}{3}\left[S O_{\text {red }}\left(\overline{O_{k}}\right)+S O_{\text {red }}\left(\overline{P_{k}}\right)+S O_{\text {red }}\left(\overline{M_{k}}\right)\right]=\left[\frac{10 \sqrt{2}}{3}+\frac{10 \sqrt{10}}{3}\right] k+\frac{4 \sqrt{2}}{3}-\frac{8 \sqrt{10}}{3} . \\
& \frac{1}{3}\left[S O_{\text {avg }}\left(\overline{O_{k}}\right)+S O_{\text {avg }}\left(\overline{P_{k}}\right)+S O_{\text {avg }}\left(\overline{M_{k}}\right)\right]=\left[\frac{22 \sqrt{2} k}{3(5 k+1)}-\frac{10 \sqrt{2}}{3(5 k+1)}+\frac{20 \sqrt{17 k^{2}+14 k+5}}{3(5 k+1)}\right] k-\frac{44 \sqrt{2}}{3(5 k+1)}+\frac{20 \sqrt{2}}{3(5 k+1)}- \\
& \frac{40 \sqrt{17 k^{2}+14 k+5}}{3(5 k+1)}+\frac{16 \sqrt{2}}{11}+\frac{8 \sqrt{101}}{11} .
\end{aligned}
$$

Thus, the average value of these indices $S O\left(\hat{\operatorname{R} S C}\left(k, \zeta_{1}, \zeta_{2}\right)\right), \operatorname{SO} O_{\text {red }}\left(\hat{\mathrm{R} S C}\left(k, \zeta_{1}, \zeta_{2}\right)\right)$ or $S O_{\text {avg }}\left(\hat{\mathrm{R} S C}\left(k, \zeta_{1}, \zeta_{2}\right)\right)$ is equal to the average value of the special chains in the set $\left\{\bar{O}_{k}, \overline{P_{k}}, \overline{M_{k}}\right\}$.

\section{7- Ratio between Sombor, Reduced Sombor and Average Sombor Index for Spiro Chain}

By getting results from theorem $(5-1,5-2)$ and (5-3), we will make the ratio between the expected values for the $S O$, $S O_{\text {red }}$ and $S O_{\text {avg }}$ with the same probabilities $\zeta_{1}$ and $\zeta_{2}$ of a random spiro-chain. 


\section{7-1-Theorem}

If $k \geq 2$, then:

$\mathrm{E}^{\mathrm{SO}}\left[\mathrm{SO}\left(\operatorname{ŔSC}\left(\mathrm{k}, \zeta_{1}, \zeta_{2}\right)\right)\right]>\mathrm{E}^{\mathrm{red}}\left[\operatorname{redSO}\left(\mathrm{R} S C\left(\mathrm{k}, \zeta_{1}, \zeta_{2}\right)\right)\right]$.

Proof: The statement is true for $k=2$. Thus for $k>2$, by applying theorem (5-1) and (5-2), we get;

$\mathrm{E}^{\mathrm{SO}}\left[\mathrm{SO}\left(\mathrm{R} S C\left(\mathrm{k}, \zeta_{1}, \zeta_{2}\right)\right)\right]-\mathrm{E}^{\mathrm{red}}\left[\operatorname{redSO}\left(\operatorname{ŔSC}\left(\mathrm{k}, \zeta_{1}, \zeta_{2}\right)\right)\right]=\left\{\left[(6 \sqrt{2}-4 \sqrt{5}) \zeta_{1}+(4 \sqrt{2}+8 \sqrt{5})\right](\mathrm{k}-2)+\right.$ $(16 \sqrt{2}+8 \sqrt{5})\}-\left\{\left[(4 \sqrt{2}-2 \sqrt{10}) \zeta_{1}+(2 \sqrt{2}+4 \sqrt{10})\right](\mathrm{k}-2)+(8 \sqrt{2}+4 \sqrt{10})\right\}$

$=\left[(2 \sqrt{2}-4 \sqrt{5}+2 \sqrt{10}) \zeta_{1}+(2 \sqrt{2}+8 \sqrt{5}-4 \sqrt{10})\right](k-2)+(8 \sqrt{2}+8 \sqrt{5}-4 \sqrt{5})$

Since;

$(2 \sqrt{2}-4 \sqrt{5}+2 \sqrt{10})>0,(2 \sqrt{2}+8 \sqrt{5}-4 \sqrt{10})>0,(8 \sqrt{2}+8 \sqrt{5}-4 \sqrt{5})>0$

So;

$=\left[(2 \sqrt{2}-4 \sqrt{5}+2 \sqrt{10}) \zeta_{1}+(2 \sqrt{2}+8 \sqrt{5}-4 \sqrt{10})\right](k-2)+(8 \sqrt{2}+8 \sqrt{5}-4 \sqrt{5})>0$,

$\because k \geq 2,0<\zeta_{1}<1$

\section{7-2-Theorem}

If $k \geq 2$, then;

$\mathrm{E}^{\mathrm{red}}\left[\operatorname{redSO}\left(\mathrm{R} S C\left(\mathrm{k}, \zeta_{1}, \zeta_{2}\right)\right)\right]>\mathrm{E}^{\mathrm{avg}}\left[\operatorname{avgSO}\left(\operatorname{ŔSC}\left(\mathrm{k}, \zeta_{1}, \zeta_{2}\right)\right)\right]$.

Proof: The statement is true for $k=2$. Thus for $k>2$, by applying theorem (5-2) and (5-3), we get:

$\mathrm{E}^{\text {red }}\left[\operatorname{redSO}\left(\operatorname{ŔSC}\left(\mathrm{k}, \zeta_{1}, \zeta_{2}\right)\right)\right]-\mathrm{E}^{\text {avg }}\left[\operatorname{avgSO}\left(\operatorname{ŔSC}\left(\mathrm{k}, \zeta_{1}, \zeta_{2}\right)\right)\right]=\left\{\left[(4 \sqrt{2}-2 \sqrt{10}) \zeta_{1}+(2 \sqrt{2}+4 \sqrt{10})\right](k-2)+\right.$ $(8 \sqrt{2}+4 \sqrt{10})\}-\left\{\left[\left(\frac{10 \sqrt{2} k+2 \sqrt{2}}{5 k+1}-\frac{4 \sqrt{17 k^{2}+14 k+5}}{5 k+1}\right) \zeta_{1}+\left(\frac{4 \sqrt{2} k-4 \sqrt{2}}{5 k+1}+\frac{8 \sqrt{17 k^{2}+14 k+5}}{5 k+1}\right)\right](k-2)+\left(\frac{16 \sqrt{2}}{11}+\frac{8 \sqrt{101}}{11}\right)\right\}$ $=\left[\left(2 \sqrt{2}-2 \sqrt{10}+\frac{4 \sqrt{17 k^{2}+14 k+5}}{5 k+1}\right) \zeta_{1}+\left(\frac{16 \sqrt{2}(k+1)}{5 k+1}+4 \sqrt{10}-\frac{8 \sqrt{17 k^{2}+14 k+5}}{5 k+1}\right)\right](k-2)+\left(\frac{72 \sqrt{2}}{11}+4 \sqrt{10}-\frac{8 \sqrt{101}}{11}\right)$ $=\left[\left(2 \sqrt{10}-\frac{4 \sqrt{17 k^{2}+14 k+5}}{5 k+1}\right)\left(2-\zeta_{1}\right)+\left(2 \sqrt{10}+\frac{16 \sqrt{2}(k+1)}{5 k+1}\right)\right](k-2)+\left(\frac{72 \sqrt{2}}{11}+4 \sqrt{10}-\frac{8 \sqrt{101}}{11}\right)$

Since;

$\left(2 \sqrt{10}-\frac{4 \sqrt{17 k^{2}+14 k+5}}{5 k+1}\right)>0,\left(2 \sqrt{10}+\frac{16 \sqrt{2}(k+1)}{5 k+1}\right)>0,\left(\frac{72 \sqrt{2}}{11}+4 \sqrt{10}-\frac{8 \sqrt{101}}{11}\right)>0, k \geq 2$

So;

$=\left[\left(2 \sqrt{10}-\frac{4 \sqrt{17 k^{2}+14 k+5}}{5 k+1}\right)\left(2-\zeta_{1}\right)+\left(2 \sqrt{10}+\frac{16 \sqrt{2}(k+1)}{5 k+1}\right)\right](k-2)+\left(\frac{72 \sqrt{2}}{11}+4 \sqrt{10}-\frac{8 \sqrt{101}}{11}\right)>0, \because k \geq 2,0<\zeta_{1}<1$

\section{7-3-Theorem}

If $k \geq 2$, then;

$\mathrm{E}^{\mathrm{SO}}\left[\mathrm{SO}\left(\hat{\mathrm{R} S C}\left(\mathrm{k}, \zeta_{1}, \zeta_{2}\right)\right)\right]>\mathrm{E}^{\mathrm{avg}}\left[\operatorname{avgSO}\left(\operatorname{ŔSC}\left(\mathrm{k}, \zeta_{1}, \zeta_{2}\right)\right)\right]$.

Proof: The statement is true for $k=2$. Thus for $k>2$, by applying theorem (5-1) and (5-3) we get;

$\mathrm{E}^{\mathrm{SO}}\left[\mathrm{SO}\left(\mathrm{R} S C\left(\mathrm{k}, \zeta_{1}, \zeta_{2}\right)\right)\right]-\mathrm{E}^{\mathrm{avg}}\left[\operatorname{avgSO}\left(\operatorname{ŔSC}\left(\mathrm{k}, \zeta_{1}, \zeta_{2}\right)\right)\right]=\left\{\left[(6 \sqrt{2}-4 \sqrt{5}) \zeta_{1}+(4 \sqrt{2}+8 \sqrt{5})\right](\mathrm{k}-2)+\right.$ $(16 \sqrt{2}+8 \sqrt{5})\}-\left\{\left[\left(\frac{10 \sqrt{2} \mathrm{k}+2 \sqrt{2}}{5 \mathrm{k}+1}-\frac{4 \sqrt{17 \mathrm{k}^{2}+14 \mathrm{k}+5}}{5 \mathrm{k}+1}\right) \zeta_{1}+\left(\frac{4 \sqrt{2} \mathrm{k}-4 \sqrt{2}}{5 \mathrm{k}+1}+\frac{8 \sqrt{17 \mathrm{k}^{2}+14 \mathrm{k}+5}}{5 \mathrm{k}+1}\right)\right](\mathrm{k}-2)+\left(\frac{16 \sqrt{2}}{11}+\frac{8 \sqrt{101}}{11}\right)\right\}$ $=\left[\left(4 \sqrt{2}-4 \sqrt{5}+\frac{4 \sqrt{17 \mathrm{k}^{2}+14 \mathrm{k}+5}}{5 \mathrm{k}+1}\right) \zeta_{1}+\left(14 \sqrt{2}\left(\frac{19 \mathrm{k}+5}{5 \mathrm{k}+1}\right)+8 \sqrt{5}-\frac{8 \sqrt{17 \mathrm{k}^{2}+14 \mathrm{k}+5}}{5 \mathrm{k}+1}\right)\right](\mathrm{k}-2)+\left(\frac{160 \sqrt{2}}{11}+8 \sqrt{5}-\frac{8 \sqrt{101}}{11}\right)$ $=\left[\left(4 \sqrt{5}-\frac{4 \sqrt{17 \mathrm{k}^{2}+14 \mathrm{k}+5}}{5 \mathrm{k}+1}\right)\left(2-\zeta_{1}\right)+\left(4 \sqrt{2}+14 \sqrt{2}\left(\frac{19 \mathrm{k}+5}{5 \mathrm{k}+1}\right)\right)\right](\mathrm{k}-2)+\left(\frac{160 \sqrt{2}}{11}+8 \sqrt{5}-\frac{8 \sqrt{101}}{11}\right)$

Since

$$
\left(4 \sqrt{5}-\frac{4 \sqrt{17 \mathrm{k}^{2}+14 \mathrm{k}+5}}{5 \mathrm{k}+1}\right)>0,\left(4 \sqrt{2}+14 \sqrt{2}\left(\frac{19 \mathrm{k}+5}{5 \mathrm{k}+1}\right)\right)>0,\left(\frac{160 \sqrt{2}}{11}+8 \sqrt{5}-\frac{8 \sqrt{101}}{11}\right)>0, \mathrm{k} \geq 2 .
$$


So;

$=\left[\left(4 \sqrt{5}-\frac{4 \sqrt{17 k^{2}+14 k+5}}{5 k+1}\right)\left(2-\zeta_{1}\right)+\left(4 \sqrt{2}+14 \sqrt{2}\left(\frac{19 k+5}{5 k+1}\right)\right)\right](k-2)+\left(\frac{160 \sqrt{2}}{11}+8 \sqrt{5}-\frac{8 \sqrt{101}}{11}\right)>0, \because k \geq 2,0<\zeta_{1}<1$.

\section{7-4-Corollary}

For $k \geq 2, \quad \mathrm{E}^{\mathrm{SO}}\left[\operatorname{SO}\left(\operatorname{ŔSC}\left(\mathrm{k}, \zeta_{1}, \zeta_{2}\right)\right)\right]>\mathrm{E}^{\mathrm{red}}\left[\operatorname{redSO}\left(\operatorname{ŔSC}\left(\mathrm{k}, \zeta_{1}, \zeta_{2}\right)\right)\right]>\mathrm{E}^{\mathrm{avg}}\left[\operatorname{avgSO}\left(\operatorname{ŔSC}\left(\mathrm{k}, \zeta_{1}, \zeta_{2}\right)\right)\right]$ as shown in Figure 6:

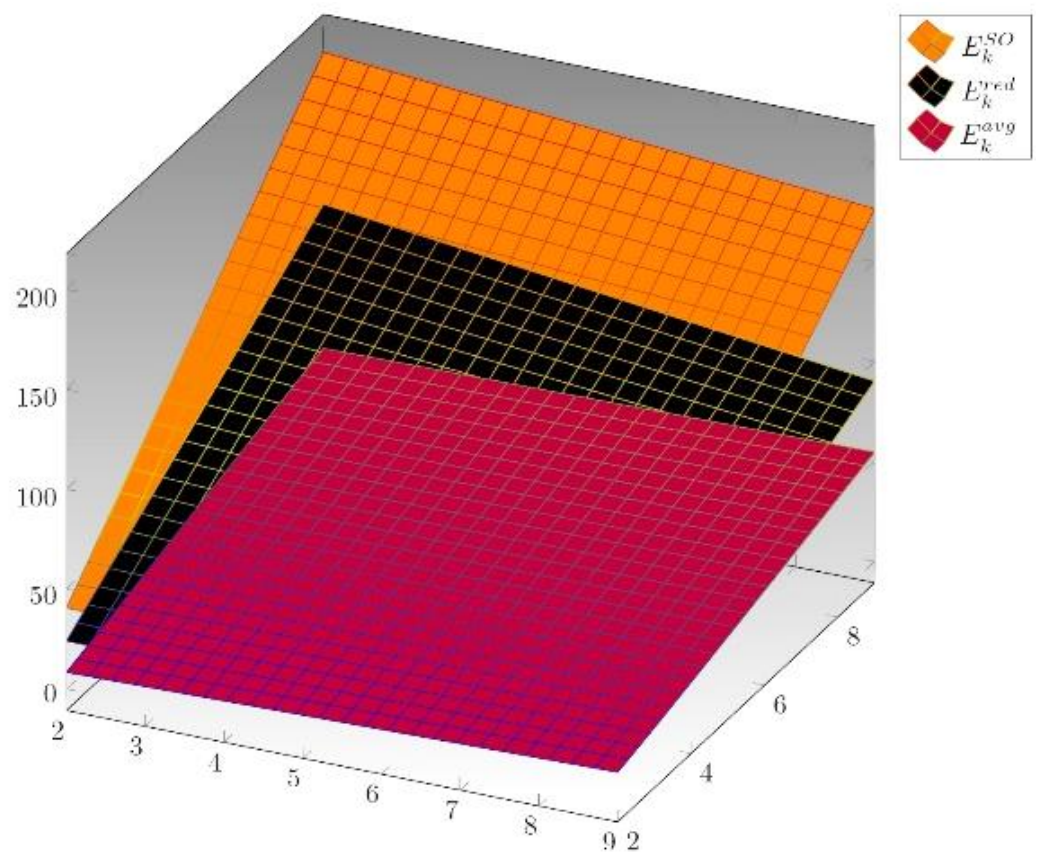

Figure 3. Comparison between the expected values of Sombor $E_{k}^{S o}$ (blue), reduced Sombor $E_{k}^{r e d}$ (red) and average Sombor $E_{k}^{a v g}$ (green) index in random spiro chains

\section{8- Conclusion}

The study of topological index is very helpful in discovering the basic topologies of different networks. Gutman introduced the SO index as a new indicator of mathematical formulation. Many topological indices are described in the literature, and many of them have been found to have applications such as physical modeling, chemical, pharmaceutical, and other molecular properties. We have discussed the $S O, S O_{\text {red }}$ and $S O_{\text {avg }}$ to find the expected value of random Polyphenyl chains and random spiro-chain. The average value of indices for both chains have been resolved. Finally, we have computed the comparison between the expected value of all Sombor indices. Figures 4 and 6 show that the expected value of the Sombor index is always greater than the other two mentioned indices. One can also deduce from it that the Sombor index is much better correlated, as compared to the other indices, with the physical properties of the compounds which are formed by these chains. Finding chemical applications for this Sombor index is an alluring task for the near future. We are also interested in building new networks and studying their geographical indications, which will greatly help to understand their priorities.

\section{9- Declarations}

\section{9-1-Author Contributions}

Conceptualization, Z.R.; methodology, Z.R.; software, K.N.; validation, Z.R., K.N. and S.A.; formal analysis, Z.R.; investigation, S.A.; resources, Z.R.; data curation, K.N.; writing—original draft preparation, Z.R.; writing-review and editing, K.N. and S.A.; visualization, K.N.; supervision, S.A.; project administration, Z.R.; funding acquisition, Z.R. All authors have read and agreed to the published version of the manuscript.

\section{9-2-Data Availability Statement}

The data presented in this study are available on request from the corresponding author. 


\section{9-3-Funding}

Zahid Raza has been supported by the University of Sharjah (UAE) under Project \#2102144098 and MASEP research group.

\section{9-4- Conflicts of Interest}

The authors declare that there is no conflict of interests regarding the publication of this manuscript. In addition, the ethical issues, including plagiarism, informed consent, misconduct, data fabrication and/or falsification, double publication and/or submission, and redundancies have been completely observed by the authors.

\section{0- References}

[1] Huang, G., Kuang, M., \& Deng, H. (2015). The expected values of Kirchhoff indices in the random polyphenyl and spiro chains. Ars Mathematica Contemporanea, 9(2), 207-217. doi:10.26493/1855-3974.458.7b0.

[2] Fang, X., You, L., \& Liu, H. (2021). The expected values of Sombor indices in random hexagonal chains, phenylene chains and Sombor indices of some chemical graphs. International Journal of Quantum Chemistry, 121(17). doi:10.1002/qua.26740.

[3] Reti, T., Dosli, T., \& Ali, A. (2021). On the Sombor index of graphs. Contributions to Mathematics, 3, 11-18. doi: $10.47443 / \mathrm{cm} .2021 .0006$.

[4] Ulker, A., Gursoy, A., Gursoy, N. K., \& Gutman, I. (2021). Relating graph energy and Sombor index. In Discrete Mathematics Letters (Vol. 8, pp. 6-9). doi:10.47443/dml.2021.0085.

[5] Bonchev, D. (1983). Information theoretic indices for characterization of chemical structures (No. 5). Research Studies Press.

[6] Deng, H. (2012). Wiener indices of spiro and polyphenyl hexagonal chains. Mathematical and Computer Modelling, 55(3-4), 634-644. doi:10.1016/j.mcm.2011.08.037.

[7] Wu, T., \& Lü, H. (2019). Hyper-Wiener indices of polyphenyl chains and polyphenyl spiders. In Open Mathematics, 17(1), , 668-676. doi:10.1515/math-2019-0053.

[8] Redžepović, I. (2021). Chemical applicability of Sombor indices. Journal of the Serbian Chemical Society, 86(5), $445-457$. doi:10.2298/JSC201215006R.

[9] Deng, H., \& Tang, Z. (2014). Kirchhoff indices of spiro and polyphenyl hexagonal chains. Utilitas Mathematica, 95, 113-128.

[10] Došlic, T., \& Litz, M. S. (2012). Matchings and independent sets in polyphenylene chains. Match-Communications in Mathematical and Computer Chemistry, 67(2), 313.

[11] Yang, Y., Liu, H., Wang, H., \& Sun, S. (2017). On Spiro and polyphenyl hexagonal chains with respect to the number of BCsubtrees. International Journal of Computer Mathematics, 94(4), 774-799. doi:10.1080/00207160.2016.1148811.

[12] Raza, Z. (2020). The Harmonic and Second Zagreb Indices in Random Polyphenyl and Spiro Chains. Polycyclic Aromatic Compounds. doi:10.1080/10406638.2020.1749089.

[13] Wang, G., Li, X., \& Li, C. (2014). Polyphenyl Systems with Extremal Hyper-Wiener Indices. Journal of Computational and Theoretical Nanoscience, 11(4), 1129-1132. https://doi.org/10.1166/jctn.2014.3472.

[14] Raza, Z. (2020). The expected values of arithmetic bond connectivity and geometric indices in random phenylene chains. Heliyon, 6(7). doi:10.1016/j.heliyon.2020.e04479.

[15] Bai, Y., Zhao, B., \& Zhao, P. (2009). Extremal merrifield-simmons index and Hosoya index of polyphenyl chains. Match, 62(3), 649-656.

[16] Chen, A., \& Zhang, F. (2009). Wiener index and perfect matchings in random phenylene chains. Match, 61(3), 623-630.

[17] Raza, Z.; Imran, M. (2021). Expected Values of Some Molecular Descriptors in Random Cyclooctane Chains. Symmetry, 13(11), 2197. doi:10.3390/sym13112197.

[18] Bian, H., \& Zhang, F. (2009). Tree-like polyphenyl systems with extremal Wiener indices. Match, 61(3), 631-642.

[19] Bureš, M., Pekárek, V., \& Ocelka, T. (2008). Thermochemical properties and relative stability of polychlorinated biphenyls. Environmental Toxicology and Pharmacology, 25(2), 148-155. doi:10.1016/j.etap.2007.10.010.

[20] Yang, Y., Liu, H., Wang, H., \& Fu, H. (2015). Subtrees of spiro and polyphenyl hexagonal chains. Applied Mathematics and Computation, 268, 547-560. doi:10.1016/j.amc.2015.06.094. 